\title{
The Rationalizability of Two-Step Choices
}

\author{
Ruth Poproski
}

May 2009

\begin{abstract}
This paper presents a formal characterization of a two stage decision rule, a version of which is presented by Isaac Levi in his book, Hard Choices. This characterization involves three conditions which, together, are satisfied by any choice function that can be represented as a two-tier choice function. And any choice function that satisfies these three conditions can be represented as a twotier choice function. The first condition - TT $\alpha$-identifies particular features of two-tier choice functions when they violate Property $\alpha$. The other two conditions - TTIC $(1)$ and TTIC $(2)$ - are essentially existence claims, required to ensure that the two tiers of a choice function can be represented without the use of cyclic orderings. My aim is to offer a formal result that provides insight into the nature of two-tier decisions. The more we understand how a particular decision procedure operates, the more we should expect to predict its results accurately, and the more we should be able to understand the circumstances under which its use is (and is not) appropriate.
\end{abstract}

\section{Introduction}

In Hard Choices, Isaac Levi presents a straightforward decision rule that runs as follows: First, narrow your choices down by choosing options that are best (i.e. maximal) according to a particular set of values. Then, assuming there is still more than one option on the table, choose from the smaller set by choosing the option that is best (i.e. maximal) according to a secondary value. Conceivably, any number of similar steps can be taken in order to reach a decision, with the range of viable options shrinking at each stage. With this process, an agent can utilize a hierarchy of values, rather than being forced to satisfy every competing constraint in one shot. 
It is reasonable to expect that a good decision rule can be characterized in terms of two types of consistency conditions: contraction and expansion. A contraction condition tells you that if you select an option $x$ from a particular set, then it should not drop out of the choice set simply because some other elements are dropped from the set. An expansion condition works in the opposite direction: if a choice rule directs you to choose an option $x$ from a particular set, then this decision should not arbitrarily change when new elements are added to the choice set. In general, the idea is that an ordering of options should not be upset simply by the addition or removal of options to or from the original set.

Interestingly, Levi's multi-tier decision rule does not satisfy the normal contraction and expansion conditions that are used to characterize rational decision making. And yet Levi's multi-tier decision rule seems like a perfectly rational sort of way to proceed.

The main purpose of this paper is to present three conditions that, taken together, characterize (a simplified version of) Levi's decision rule. It turns out that contraction and expansion consistency is not at the center of this characterization. Rather, the key to understanding the formalized nature of two-tiered decision rules lies in the interaction between the tiers, as sets of options change.

In Sections 2 and 3 below I introduce the version of Levi's multi-tier decision procedure that I aim to characterize in this paper. In Section 4 I present typical contraction and expansion conditions, and I briefly discuss how they are (and are not) relevant for two-tier choice functions. As it turns out, contraction inconsistency, not consistency, plays a role in my characterization of two-tier choice functions. The main result of my paper is in Section 5. There I provide a characterization of two-tier choice functions in terms of three conditions, which I call TT $\alpha$, TTIC(1), and $\operatorname{TTIC}(2)$.

My aim is not to provide an argument for the rational nature of multi-tier decisions (although I think one can be made), nor to defend a particular approach to the representation of preferences. Rather, I offer a formal result that provides insight into the nature of two-tier decisions. The more we understand how a particular decision procedure operates, the more we should expect to predict its results accurately, and the more we should be able to understand the circumstances under which its use is (and is not) appropriate.

\section{Multi-Tiered Decisions}

The prototypical example of a multi-tier decision process is that of hiring an individual to fill a job. In the first tier of the decision, the pool of applicants is narrowed down 
by considering a variety of factors: previous work experience, specific skills, reports from references, and so on. Each of these factors can be treated as a value, and the candidates can be ranked against one another with respect to each of these values. The candidates who make it past the first tier of this decision process will be those who are thought to be 'best, all things considered'.

Exactly what it means to be 'best, all things considered' can vary from situation to situation. One option is to select any candidate who is better than all the rest with respect to at least one of the preference relations: she is optimal (or maximal, if the rankings are not complete) with respect to at least one value. Alternatively, if we take the cardinal approach, one might aggregate various scores (according to some acceptable scoring method), and select any candidate whose aggregate score is above a certain threshold. Some versions of this approach will make way for a candidate who is never best, but always second best, to be included in the set of candidates who are 'best, all things considered'.

Either way, the first tier of the decision procedure involves several preference relations, and will likely leave the decision-making agent with a variety of candidates to choose between.

As you might suspect, the second tier of the decision procedure will involve more preference rankings. And again, the number of candidates who might be hired will (hopefully) decrease. If more than one candidate remains, yet another tier will be added to the mix, and yet more values will be considered. And we can continue this procedure until a single candidate remains, and a hiring decision is made.

Formally, we can model this decision procedure by defining a choice function for each tier. $C_{1}$ chooses from among a set of candidates according to some set of preference relations (either ordinal or cardinal) that represent the decision agent's preferences over the first-tier values. $C_{2}$ is defined in a similar fashion, but the relevant preference rankings are those that represent second-tier values. And so on, up through the tiers.

Suppose there are three tiers to the decision. Then we define the choice function $C$, for a set $S$ of candidates, as

$$
C(S)=C_{3}\left(C_{2}\left(C_{1}(S)\right)\right)
$$

\section{Two-Tier Admissibility}

The version of Levi's multi-tier decision procedure being considered in this paper is comparatively modest: I am limiting my investigation to two-tier decisions where 
there is a collection of ordinal preference rankings in the first tier, and a single, ordinal preference ranking in the second tier.

The main reason for these limitations is simplicity: This paper is a first step toward modelling multi-tiered decisions with multiple cardinal preference rankings in each tier. Identifying the details for the simple case should provide some of the machinery and insight required to model the more complex versions of multi-tiered decisions.

That said, let $U$ be a finite set of live options for a given decision problem.

Let $V_{1}(U)$ be the first-tier value structure for that decision problem. $V_{1}(U)$ consists in a series of preference relations, each of which is meant to be transitive, acyclic and complete with respect to the elements of $U$.

Let $V_{2}(U)$ be the second-tier value structure for a given decision problem. $V_{2}(U)$ consists in a single preference relation. That preference relation is meant to be transitive, acyclic and complete with respect to the elements of $U$.

Let $C_{1}$ be a choice function that picks out first-tier admissible elements by choosing any and all elements that are optimal in a set $S \subseteq U$ according to at least one first-tier ordering.

$$
C_{1}(S)=\left\{x \in S \mid \exists \succeq_{i} \in V_{1}(U) \forall y \in S: x \succeq_{i} y\right\}
$$

Let $C_{2}$ be a choice function that picks out second-tier admissible elements by choosing elements that are optimal in a set $S \subseteq U$ according to the second-tier ordering $\succeq_{2}$.

$$
C_{2}(S)=\left\{x \in S \mid \forall y \in S: x \succeq_{2} y\right\}
$$

Let $C$ be a choice function defined over $U$. If $C$ is two-tier rationalizable then, $\forall S \subseteq U$ :

$$
C(S)=C_{2}\left(C_{1}(S)\right)
$$

We say that an element $x$ is first-tier admissible in $S$ if $x \in C_{1}(S)$. We say that $x$ is second-tier admissible in $S$ if $x \in C_{2}\left(C_{1}(S)\right)$.

The purpose of this paper is to investigate the nature of two-tier rationalizable choice functions, and to offer a characterization of such functions in terms of three conditions - which I call TT $\alpha$, TTIC(1), and TTIC(2).

But before I get to that, some more background is in order. 


\section{Consistency Conditions: $\alpha, \beta, \gamma$}

Entrenched in the literature on rational decision procedures are a series of consistency conditions that seem to capture at least part of what we are aiming for in any attempt to model rational decisions.

For instance, Properties $\alpha, \beta$ and $\gamma$ are oft-cited conditions of rationality. Property $\alpha$, also known as Chernof $f^{1}$, is a contraction consistency condition. The main idea is that if an alternative is removed from a set $S$ of live options, its removal should not upset the manner in which the other alternatives are ordered. Perhaps more precisely, Property $\alpha$ states that if an option is removed from a set $S$, the output of the choice function should not be radically altered. At worst, the removal of a 'best' option should simply elevate the 'second best' option to first place.

Formally we can define Property $\alpha$ as follows:

Definition 1. Property $\alpha$

$$
\text { If } x \in C(S) \text { and } x \in T \subseteq S \text { [for some } S \subseteq U \text { ] then } x \in C(T) \text {. }
$$

An expansion consistency condition works in the opposite direction: when a new option is introduced into a set, the choice function and the orderings underlying it should not be radically altered.

Property $\beta$ is a basic expansion condition: ${ }^{2}$

Definition 2. Property $\beta$

$$
\text { If } x, y \in C(T), T \subseteq S \text { and } x \in C(S) \text { [for some } S \subseteq U \text { ] then } y \in C(S)
$$

That is, if two options are chosen from among the elements of $T$, then the addition of new options to the mix should not cause $C$ to treat the original two options differently. Another common expansion condition is Property $\gamma,{ }^{3}$ put here in its finite form:

\section{Definition 3. Property $\gamma$}

$$
\text { For any } S, T \subseteq U \text {, if } x \in C(S) \cap C(T) \text { then } x \in C(S \cup T) \text {. }
$$

\footnotetext{
${ }^{1}$ So-called because it originated with Chernoff (1954)

${ }^{2}$ For an early use of Property $\beta$ see Sen (1969)

${ }^{3}$ cf. Sen (1971)
} 


\subsection{Characterizing Single-Tiered Decisions}

Moulin (1985) draws together a number of results regarding consistency conditions for choice functions. Of particular relevance for this paper are his second and fifth theorems, which characterize choice functions that select the maximal option(s) in a set, according to some ordinal preference ranking(s).

Clearly, if we have a well-defined preference relation, we can pick the element that is maximal according to that relation. Moulin (1985) asks the converse question: "can we detect [the] existence of an underlying preference relation from which our choice function is derived"? (p. 147) That is, given an agent's choice function, can we determine the structure behind her choice? If an underlying preference ranking exists, we say that it rationalizes her choice function: we say that a choice function is rationalizable if it is characterized in terms of a single binary relation - like $\succeq_{i}-$ such that it admits only the maximal elements (according to $\succeq_{i}$ ) from a set of options.

The main result reported by Moulin regarding rationalizable choice functions is his Theorem $2:^{4}$

Moulin's Theorem 2: A choice function is rationalizable if and only if it satisfies Chernoff and Expansion.

Chernoff is just the contraction consistency condition $\alpha$ presented earlier in this paper, and Expansion is the expansion consistency condition $\gamma$, also presented earlier.

A more interesting notion is that of pseudo-rationalizability. A choice function is pseudo-rationalizable if it selects those options that are maximal according to at least one preference relation, from a set of options. That is, we say that a choice function is pseudo-rationalizable if it is characterized in terms of one or more preference relations and admits only the elements that are maximal according to at least one of those relations.

Moulin characterizes pseudo-rationalizability in terms of the contraction condition Chernoff $(\alpha)$, and an expansion condition that gained prominence in the literature due to work by Mark Aizerman: ${ }^{5}$

\section{Definition 4. Aizerman:}

$$
\text { If } C(S) \subseteq T \subseteq S \text { [for some } S \subseteq U] \text { then } C(T) \subseteq C(S)
$$

\footnotetext{
${ }^{4}$ credited to Sen (1971)

${ }^{5}$ cf. Aizerman \& Malishevsky (1981)
} 
Moulin's Theorem 5 reads as follows:

Moulin's Theorem 5: A choice function is pseudo-rationalizable if and only if it satisfies Chernoff and Aizerman.

The relevance of these results to the current project is relatively straightforward: in the version of two-tier admissibility being considered in this paper, the first tier choice function $C_{1}$ is pseudo-rationalizable, and the second tier choice function $C_{2}$ is rationalizable. In other words, the individual tiers of multi-tiered decisions are well-behaved.

\subsection{Consistency Conditions and Two-Tier Admissibility}

One of the interesting features of Levi's two-tier decision rule is that, even in the simple form under consideration in this paper, it does not satisfy Property $\alpha$, Property $\beta$, or Property $\gamma$. Each of these properties seems, on the face of it, like the sort of property that should characterize rational decision procedures. And Amartya Sen, among others, has gone to great lengths to characterize choice functions, revealed preference, and rationality in terms of adherence to Properties $\alpha, \beta$ and $\gamma$.

But Levi's multi-tier decision rule is also, on the face of it, a rational decision procedure. One might want to debate the variety of approaches taken to representing preferences within the tiers, and the consequences each has for a multi-tiered decision rule, but the fact that Levi's rule does not satisfy standard properties like $\alpha, \beta$, and $\gamma$ is not fodder for its immediate dismissal. The general idea of decision-making in distinct stages is, at least on the face of it, a rational way to proceed.

A natural question arising from this failure of $\alpha, \beta$ and $\gamma$ is as to what sorts of conditions Levi's multi-tier decision rule does adhere to. What happens, for instance, when elements are added to or removed from a set? How does a multi-tier choice function behave?

A further question to ask is about what conditions characterize multi-tier decisions. Is there some condition, or some set of conditions, that fully characterizes multi-tier choice functions? That is, is there any (set of) condition(s) such that a choice function is a multi-tier choice function if and only if it satisfies that (set of) condition(s)?

The main purpose of this paper is to provide a window into the characterization of my two-tier version of the rule by way of three conditions. The first condition identifies some of the consequences for two-tier choice functions when Property $\alpha$ is violated. The other two are closely related to one another and turn on the interplay between 
the two tiers - on the specifics of the orderings as they must be set up if a given choice function is to be represented in two tiers.

Each tier contains preference rankings over the elements of a set $U$. As a choice function operates over larger or smaller sets of options, different sets of elements are promoted from the first stage of the decision procedure to the second. So while each tier satisfies Property $\alpha$, the combined decision procedure does not. When the set of options is changed, $C_{1}$ generates a new set, and $C_{2}$ operates on that new set. How these two tiers interact - how the changes in the set generated by $C_{1}$ affect the results of applying $C_{2}$ - is at the center of the conditions required to characterize two-tier choice functions.

The conditions that characterize two-tier choice functions are not particularly intuitive, nor do they immediately make you believe that the decision procedure being characterized must be rational (in some sense of that word). In this way they differ radically from properties like $\alpha, \beta$ and $\gamma$. Nevertheless, they are satisfied by two-tier choice functions, and any choice function that satisfies the conditions can be represented as a two-tier choice function. And while the failure of contraction consistency plays a role in the characterization of two-tier admissibility, the main issue is not contraction or expansion consistency, but the interaction between the two tiers.

\section{Characterizing Two-Tier Admissibility}

As mentioned above, the purpose of this paper is to offer a characterization of two-tier admissibility: to characterize two-tier rationalizable choice functions. In what follows I offer a proof of the following theorem (with details of its content to be filled in as we proceed):

Theorem 1. $C$ is two-tier rationalizable if and only if $C$ satisfies conditions TT $\alpha$, $\operatorname{TTIC}(1)$, and TTIC(2).

In the forward direction, the proof is relatively straightforward. However, I will leave this part of the proof until later in order to present the conditions in a context that may reveal some of the motivation behind their use.

In the reverse direction, I will first detail a principled method of construction for two tiers $-V_{1}(U)$ and $V_{2}(U)$ - given a choice function $C$ that is defined over $U$. There are eight construction rules in total, each of which addresses a particular feature of two-tier admissibility. When applied, one after the other, these rules can produce 
$V_{1}(U)$ and $V_{2}(U)$ in such a way that the original choice function can be recovered from the constructed orderings.

It turns out that three conditions are required for successful application of the seven construction rules: a condition surrounding the failure of Property $\alpha$, called TT $\alpha$, and two iterating conditions that I call TTIC(1) and TTIC(2). I will present these conditions in the context of my construction rules, and I will show that if these construction rules are successfully applied to a choice function $C$, then $C$ is two-tier rationalizable. That is, $\forall S \subseteq U: C(S)=C_{2}\left(C_{1}(S)\right)$.

\subsection{Properties of Two-Tier Choice Functions}

There are several properties of two-tier choice functions that are particularly relevant to the project at hand. These properties will guide the two-tier construction method presented below, and therefore deserve some discussion here.

\section{Two-Tier Properties}

TTP 1. $\forall x, y \in U:$ If $I(\{x, y\})$ then $x \sim_{2} y .^{6}$

TTP 2. $\forall T \subseteq U:$ If $x \in T$ and $\forall y \in C(T) x \in C(\{x, y\})$, then $x \succeq_{2} C(T)$.

TTP 3. $\forall x \in U:$ If $T \subseteq S$ [for some $T, S \subseteq U$ ] and $x \in C(S) \cap T \backslash C(T)$, then $C(T) \succ_{2} x$.

TTP 4. $C(S) \subseteq C_{1}(S)$ [for all $\left.S \subseteq U\right]$.

TTP 5. $\forall T \subseteq U$ : If $x \in T \backslash C(T)$ and $x \succeq_{2} C(T)$, then $x \notin C_{1}(T)$.

TTP 6. The orderings are transitive.

TTP 7. The orderings are complete.

\section{Two-Tier Property 1}

To understand TTP1 some explanation is required. First, a definition of $I(\cdot)$ is in order:

Definition 5. $I(\{x, y\})$ holds if and only if there is a series of sets $T_{1}, \ldots, T_{n} \subseteq U$ $[n \geq 2]$ such that $\left\{x, z_{1}\right\} \subseteq C\left(T_{1}\right),\left\{z_{1}, z_{2}\right\} \subseteq C\left(T_{2}\right), \ldots,\left\{z_{n-2}, z_{n-1}\right\} \subseteq C\left(T_{n-1}\right)$, and $\left\{z_{n-1}, y\right\} \subseteq C\left(T_{n}\right)$ [for some $x, y, z_{1}, \ldots, z_{n-1} \in U$ ].

\footnotetext{
${ }^{6} I(\cdot)$ is defined below.
} 
This relation is reflexive and transitive.

Definition 6. $I(T)$ holds if and only if $I(\{x, y\})$ holds for every $\{x, y\} \subseteq T$.

TTP1 amounts to a claim that the following lemma is true:

Lemma 1. If $C$ is a two-tier choice function defined over $U$ and $I(\{x, y\})$ holds [for some $x, y \in U]$, then $x \sim_{2} y$.

Proof. Let $C$ be a two-tier choice function defined over $U$, let $a, b \in U$, and suppose $I(\{a, b\})$. Then there is a series of sets $T_{1}, \ldots, T_{n} \subseteq U$ and a series of elements $z_{1}, \ldots, z_{n-1} \in U$ such that $\left\{a, z_{1}\right\} \subseteq C\left(T_{1}\right),\left\{z_{1}, z_{2}\right\} \subseteq C\left(T_{2}\right), \ldots,\left\{z_{n-1}, b\right\} \subseteq C\left(T_{n}\right)$. Now for any two elements, $\{x, y\} \subseteq C\left(T_{i}\right)$, it is also the case the $\{x, y\} \subseteq C_{1}\left(T_{i}\right)$; and if $\{x, y\} \subseteq C_{1}\left(T_{i}\right)$ and $x \succ_{2} y$, then $y \notin C_{2}\left(C_{1}\left(T_{i}\right)\right)$. So, if $\{x, y\} \subseteq C\left(T_{i}\right)$ then $\neg\left(x \succ_{2} y\right)$ and $\neg\left(y \succ_{2} x\right)$. That is, $y \succeq_{2} x$ and $x \succeq_{2} y$ (since the ordering is complete). Which is just to say that $x \sim_{2} y$. And so, since $I(\{a, b\})$ holds we know that $a \sim_{2} z_{1}, z_{1} \sim_{2} z_{2}, \ldots, z_{n-1} \sim_{2} b$. And by the transitivity of $\sim_{2}$ it follows that $a \sim \sim_{2} b$.

\section{Two-Tier Property 2}

The second property is slightly less obvious, and a quick lemma is in order to show that it is true:

Lemma 2. If $C$ is a two-tier choice function, $x \in T \subseteq U$, and $\forall y \in C(T) x \in$ $C(\{x, y\})$, then $x \succeq_{2} C(T)$.

Proof. Suppose $C$ is a two-tier choice function defined over $U$, let $a \in T \subseteq U$, and suppose that $\forall x \in C(T) a \in C(\{a, x\})$. If $a \in C(T)$ then $a \sim_{2} C(T)$, and hence $a \succeq_{2} C(T)$. Suppose instead that $a \notin C(T)$ and, without loss of generality, let $b \in C(T)$. Then, since $\forall x \in C(T) a \in C(\{a, x\})$, it follows that $a \in C(\{a, b\})$. So either $a \succeq_{2} b$ or $b$ is not first-tier admissible in $\{a, b\}$ (otherwise, $a \notin C_{2}\left(C_{1}(\{a, b\})\right)$ ). If $b \notin C_{1}(\{a, b\})$ then, for every first-tier ordering $\succeq_{i}, a \succ_{i} b$. But $a \in T$, so this would mean that $b \notin C_{1}(T)$, and hence $b \notin C(T)$, contrary to supposition. So $b \in C_{1}(\{a, b\})$ and hence $a \succeq_{2} b$. Now since $b \in C(T)$ we have that $b \sim_{2} C(T)$. By the transitivity of $\succeq_{2}$, then, $a \succeq_{2} C(T)$. And so, if $C$ is a two-tier choice function, $a \in T \subseteq U$, and $\forall x \in C(T) a \in C(\{a, x\})$, then $a \succeq_{2} C(T)$.

\section{Two-Tier Property 3}

Notice that TTP3 is relevant any time $\mathrm{C}$ violates Property $\alpha$. 
Lemma 3. If $C$ is a two-tier choice function, $T \subseteq S$ [for some $S \subseteq U]$ and $x \in$ $C(S) \cap T \backslash C(T)$, then $C(T) \succ_{2} x$.

The proof of this lemma is quick and easy:

Proof. Suppose $C$ is a two-tier choice function, $T \subseteq S \subseteq U$ and $a \in C(S) \cap T \backslash C(T)$. Then $a \in C_{1}(S)$. And since $C_{1}$ satisfies Property $\alpha$ and $T \subseteq S$, then $a \in C_{1}(T)$. But $a \notin C(T)$ so it must be the case that $C(T) \succ_{2} a$.

These three two-tier properties are the main features of two-tier choice functions that are necessary for the construction of $V_{2}(U)$, according to my set-up below. I will expand on this claim later, but first let us continue to examine the proposed properties of two-tier choice functions.

\section{Two-Tier Property 4}

The fourth property, TTP4, is straightforward. For any well-formed choice function $C, C(S) \subseteq S$ : the set of selected elements is a subset of the set from which it is selecting. And since $C$ is a two-tier choice function if and only if $C(S)=C_{2}\left(C_{1}(S)\right)$, and $C_{1}$ is a well-formed choice function, it naturally follows that $C(S) \subseteq C_{1}(S)$.

\section{Two-Tier Property 5}

This property states that if some element $x$ of $T$ is not in $C(T)$ then either the elements of $C(T)$ strictly dominate $x$ in the second tier, or $x$ is not first-tier admissible in $T$.

Lemma 4. If $C$ is a two-tier choice function, $x \in T \backslash C(T)$ and $x \succeq_{2} C(T)$ [for some $T \subseteq U]$ then $x \notin C_{1}(T)$.

The proof is straightforward, but is included here for completeness' sake.

Proof. Suppose $C$ is a two-tier choice function defined over $U$, let $a \in T \backslash C(T)[T \subseteq U]$ and suppose $a \succeq_{2} C(T)$. If $a \in C_{1}(T)$ then either $a \in C_{2}\left(C_{1}(T)\right)$ or there is some $x \in C_{1}(T)$ such that $x \succ_{2} a$. Suppose the latter: let $b \in C_{1}(T)$ and suppose $b \succ_{2} a$. So, by the transitivity of $\succeq_{2}, b \succ_{2} C(T)$; and hence $\forall x \in C(T), x \notin C_{2}\left(C_{1}(T)\right)$. So if $a \in C_{1}(T)$ then either $a \in C_{2}\left(C_{1}(T)\right) \backslash C(T)$ or $\forall x \in C(T), x \notin C_{2}\left(C_{1}(T)\right)$. But $C$ is a two-tier choice function, so $C(T)=C_{2}\left(C_{1}(T)\right)$. And hence $a \notin C_{1}(T)$.

This property turns out to be the key to unlocking a characterization of two-tier admissibility. The distinctive feature of a two-tier choice function is not its failure of Properties $\alpha, \beta$ and $\gamma$, but the manner in which $C$ selects over successive tiers. TTP5 gets at the interaction between the tiers for a two-tier choice function, and it 
will play a crucial role in the construction of $V_{1}(U)$ and $V_{2}(U)$ from a choice function C.

Notice, however, that this property of choice functions can be addressed in either the construction of $V_{1}(U)$ or the construction of $V_{2}(U)$. If the latter approach is taken, then the first-tier orderings will need to be completed first, and then the second-tier orderings can be constructed such that if $x \in T \backslash C(T)$ and $x \in C_{1}(T)$ then $C(T) \succ_{2} x$. Alternatively, we can construct $V_{2}(U)$ first, and then, if $x \in T \backslash C(T)$ and $x \succeq_{2} C(T)$, we can construct $V_{1}(U)$ such that $x \notin C_{1}(T)$. For reasons discussed below, I will incorporate TTP5 into my construction method by first (partially) constructing $\succeq_{2}$, and then constraining the orderings in $V_{1}(U)$ to ensure that if $x \in T \backslash C(T)$ and $x \succeq_{2} C(T)$, then $x \notin C_{1}(T)$.

\section{Transitivity and Completeness}

The final two properties - TTP6 (transitivity) and TTP7 (completeness) - are really just reminders of what it means to be a well-formed ordering. In the version of two-tier admissibility being considered in this paper, every ordering is meant to be transitive - in both its strict and its weak parts. The construction rules detailed below will incorporate this property into the orderings that are built.

The orderings associated with a two-tier choice function need not be complete, but it simplifies matters considerably if they are. Without completeness it could turn out that $C(S)$ is undefined for some sets $S \subseteq U$. Dealing with this complication goes beyond the scope of this paper, and so I will incorporate completeness into my construction of the orderings $V_{1}(U)$ and $V_{2}(U)$.

\subsection{Constructing the Second Tier}

The first step in our construction is to establish a basic framework for $V_{2}(U)$. Specifically, we need to ensure that TTP1, TTP2, and TTP3 are satisfied by $V_{2}(U)$. To this end, the following definition will be useful:

Definition 7. $G(x, y)$ if and only if

(1) $I(\{x, y\})$, or

(2) $\exists T \subseteq U$ such that $x \in T, \forall z \in C(T) x \in C(\{x, z\})$, and $I(\{y\} \cup C(T))$.

For any two-tier choice function, if $G(x, y)$ holds then - by Lemmas 1 and 2 and the transitivity of $\succeq_{2}-x \succeq_{2} y$. Notice also that $G(x, x)$ holds, for any $x$, because 
$I(\{x, x\})$ always holds. ${ }^{7}$ Since $\succeq_{2}$ is transitive, it will also be helpful to have the following:

Definition 8. $G_{T}(x, y)$ if and only if $\exists z_{1}, \ldots, z_{n}[n \geq 1]$ such that $G\left(x, z_{1}\right), G\left(z_{1}, z_{2}\right)$, $\ldots, G\left(z_{n-1}, z_{n}\right)$, and $G\left(z_{n}, y\right)$.

Since $G(x, x)$ holds for all $x$, then $G(x, y)$ implies $G_{T}(x, y)$. In addition, we say that $G_{T}(x, T)$ holds if and only if there is some $y$ such that $G_{T}(x, y)$ and $I(\{y\} \cup T)$; and $G_{T}(T, x)$ holds if and only if $G_{T}(y, x)$ and $I(\{y\} \cup T)$.

With this machinery in hand, our first construction rule is straightforward:

CR 1. $\forall<x, y>\in U^{2}$ : If $G_{T}(x, y)$ then set $x \succeq_{2} y$.

This rule ensures that TTP1 and TTP2 are satisfied by our constructed orderings.

To deal with TTP3 a second family of relations should be defined:

Definition 9. $S(C(T), x)$ if and only if $\exists S$ such that $T \subseteq S$ and $x \in C(S) \cap T \backslash C(T)$.

By Lemma 3, if $C$ is a two-tier choice function and $S(C(T), x)$ then $C(T) \succ_{2} x$. And again, since $\succeq_{2}$ is transitive, the following will be helpful:

Definition 10. $S_{T}(C(T), x)$ if and only if $\exists y$ such that $S(C(T), y)$ and $G_{T}(y, x)$.

If $C$ is a two-tier choice function and $S_{T}(C(T), x)$ holds then, by Lemmas 1-3 and the transitivity of $\succeq_{2}$, there is some $y \in U$ such that $C(T) \succ_{2} y$ and $y \succeq_{2} x$. Since $\succeq_{2}$ is transitive, it follows that $C(T) \succ_{2} x$.

With this in hand, our second construction rule is:

CR 2. $\forall x \in U, \forall T \subseteq U$ : If $S_{T}(C(T), x)$ then set $C(T) \succ_{2} x$.

Notice that $G_{T}$ is transitive: if $G_{T}(x, y)$ and $G_{T}(y, z)$ hold then so does $G_{T}(x, z)$. Also, if $S_{T}(C(T), x)$ and $G_{T}(x, y)$ hold then so does $S_{T}(C(T), y) .{ }^{8}$ Regardless, it will be useful to close the ordering, as so far constructed, under transitivity:

CR 3. $\forall x, y, z \in U$ :

$$
\begin{aligned}
& \text { If } x \succeq_{2} y \text { and } y \succeq_{2} z \text { then set } x \succeq_{2} z . \\
& \text { If } x \succ_{2} y \text { and } y \succeq_{2} z \text { then set } x \succ_{2} z . \\
& \text { If } x \succeq_{2} y \text { and } y \succ_{2} z \text { then set } x \succ_{2} z .
\end{aligned}
$$

\footnotetext{
${ }^{7}$ Here we need to be a little generous with the notation, but the idea is that $I(\{x, x\})$ holds because $x \in C(\{x\}) \equiv C(\{x, x\})$.

${ }^{8}$ Suppose $S_{T}(C(T), a)$ and $G_{T}(a, b)$. Then there is some $c$ such that $S(C(T), c)$ and $G_{T}(c, a)$. Since $G_{T}$ is transitive then $S_{T}(C(T), a)$ and $G_{T}(a, b)$ implies that there is some $c$ such that $S(C(T), c)$ and $G_{T}(c, b)$. And hence, by definition, $S_{T}(C(T), b)$.
} 


\subsubsection{Guarding Against Cycles}

Also notice that CR1-CR3 might produce strict cycles in the ordering if they can conspire to set either $x \succ_{2} x$ or $C(T) \succ_{2} C(T)$ [for some element $x$ or set $T$ ]. The following condition, if satisfied by $C$, will prevent such problems:

Proposed Condition 1. TT $\alpha$ For any $x, y \in U$ and $T, S \subseteq U$ :

(a) If $S_{T}(C(T), x)$ then $\neg G_{T}(x, C(T))$.

(b) If $S_{T}(C(T), x), S_{T}(C(S), y)$, and $G_{T}(x, C(S))$ then $\neg G_{T}(y, C(T))$.

This condition is called ' $\mathrm{TT} \alpha$ ' because of its relation to Property $\alpha$ in two tiers. Specifically, TT $\alpha$ identifies certain consequences that arise for two-tier choice functions when Property $\alpha$ is violated. A full proof of this will be given later, but for the moment recall the definition of $S_{T}(C(T), x): S_{T}(C(T), x)$ if and only if, for some $T^{\prime}$ and $y, S\left(C\left(T^{\prime}\right), y\right)$ and $G_{T}(y, x)$. And, more importantly, $S\left(C\left(T^{\prime}\right), y\right)$ holds if and only if $y \in C(S) \cap T \backslash C(T)$, where $T \subseteq S$. So when $S_{T}(C(T), x)$ holds, Property $\alpha$ has been violated.

In terms of TT $\alpha$ 's connection to the construction of acyclic orderings, the following lemma is in order:

Lemma 5. If CR1-CR3 are used to construct $\succeq_{2}$ and $C$ satisfies $T T \alpha$, then $\succeq_{2}$ will be acyclic.

Proof. Suppose CR1-CR3 are used to construct $\succeq_{2}$. We can think of the potential cycles as taking the form $x \succ_{2} x$ for some $x \in U$, or $C(T) \succ_{2} C(T)$ for some $T \subseteq U$. Give the nature of the three construction rules, $x \succ_{2} x$ will be constructed by CR1-CR3 if and only if $x \succeq_{2} C(T)$ and $C(T) \succ_{2} x$ are constructed. That is, strict relations arise with CR2, so it must be part of the equation; and the route to seeing an element dominate a choice set must involve CR1. Which is just to say that $x \succ_{2} x$ will occur if and only if $G_{T}\left(x, C(T)\right.$ ) and $S_{T}(C(T), x)$ (recall that $G_{T}$ is transitive and if $S_{T}(C(T), y)$ and $G_{T}(y, x)$ hold, then so does $\left.S_{T}(C(T), x)\right)$. By TT $\alpha$, if $S_{T}(C(T), x)$ then $\neg G_{T}(x, C(T))$. So if TT $\alpha$ is satisfied by $C$ then CR1-CR3 will not construct $\succeq_{2}$ such that $x \succ_{2} x$ (for any $x \in U$ ).

Notice also that if $x \succeq_{2} C(T)$ and $C(T) \succ_{2} x$ are constructed then, by CR3, $C(T) \succ_{2}$ $C(T)$. But once again this is prevented by TT $\alpha$. Alternatively, $C(T) \succ_{2} C(T)$ will be constructed if we first construct $C(T) \succeq_{2} y \succeq_{2} C\left(T^{\prime}\right) \succ_{2} z \succeq_{2} C(T)$ [for some $y, z \in U$ and $\left.T^{\prime} \subseteq U\right]$. Again taking into account the nature of the construction rules and the 
various transitivity relations, this will occur if and only if $G_{T}(C(T), y), G_{T}\left(y, C\left(T^{\prime}\right)\right)$, $S_{T}\left(C\left(T^{\prime}\right), z\right)$, and $G_{T}(z, C(T))$. Now $S_{T}\left(C\left(T^{\prime}\right), z\right)$ holds if and only if, for some $w, S\left(C\left(T^{\prime}\right), w\right)$ - which implies $S_{T}\left(C\left(T^{\prime}\right), w\right)-$ and $G_{T}(w, z)$. So $C(T) \succeq_{2} y \succeq_{2}$ $C\left(T^{\prime}\right) \succ_{2} z \succeq_{2} C(T)$ will occur if and only if $S_{T}\left(C\left(T^{\prime}\right), w\right)$ and (by the transitivity of $\left.G_{T}\right) G_{T}\left(w, C\left(T^{\prime}\right)\right)$. Which is a violation of TT $\alpha$.

Given the transitivity relations for $G_{T}$ and $S_{T}$, as well as the manner in which CR1 and CR2 establish $\succeq_{2}$, we can conclude that all potential cycles produced by CR1CR3 can be reduced to the situations described above. And so we conclude: If CR1-CR3 are used to construct $\succeq_{2}$ and $C$ satisfies TT $\alpha$, then the constructed $\succeq_{2}$ will be acyclic.

\subsubsection{Dealing with TTP5}

So CR1-CR3 set up a framework for $V_{2}(U)$. However, this is not the end of the story. Obviously, we need a construction rule to complete the ordering. This is nonproblematic and will come later. But recall the fifth property on our list of important two-tier properties:

TTP 5. $\forall T \subseteq U$ : If $x \in T \backslash C(T)$ and $x \succeq_{2} C(T)$ then $x \notin C_{1}(T)$.

Somehow, our construction rules need to ensure that TTP5 is satisfied by our constructed orderings. We might begin by constructing $V_{1}(U)$ and then suggesting the following second-tier construction rule:

$$
\forall T \subseteq U: \text { If } x \in C_{1}(T) \backslash C(T) \text { then set } C(T) \succ_{2} x
$$

Unfortunately, in the presence of CR1 this will not do.

Example 1. Suppose $a \in U, b \in T \subseteq U$, and $G_{T}(a, C(T))$. By CR1 we construct $\succeq_{2}$ such that $a \succeq_{2} C(T)$. But suppose $x \in C_{1}(T) \backslash C(T)$ - perhaps because $x \in C(S)$ and $T \subseteq S .{ }^{9}$ Then, by the suggested construction rule, we are also to set $C(T) \succ_{2} a$. And hence we will have $C(T) \succ_{2} a \succeq_{2} C(T)$, which is clearly undesirable.

And CR1 is required. By Lemmas 1 and 2 and the transitivity of $\succeq_{2}$, if $C$ is a two-tier choice function and $G_{T}(x, y)$, then $x \succeq_{2} y$. Our constructed $\succeq_{2}$ must adhere to this feature if we are to have any hope of recovering $C$ from the construction. So the solution is not to give up some or all of CR1.

\footnotetext{
${ }^{9}$ Our first-tier construction must ensure that if $x \in C(S)$ then $x \in C_{1}(S)$ - to satisfy TTP4 from above. But since $C_{1}$ satisfies Property $\alpha, x \in C_{1}(S)$ implies $x \in C_{1}(T)$ for any $T \subseteq S$.
} 
In other words, TTP5 must be addressed in the construction of our first tier. The general idea behind the relevant construction rule will be that if $x \in T \backslash C(T)$ and $x \succeq_{2} C(T)$ then we should ensure that $x \notin C_{1}(T)$ - by picking some appropriate $y \in T \backslash\{x\}$ (for each first-tier ordering $\succeq_{S}$ ) and setting $y \succ_{S} x$.

These details will be provided below, but the important point at this juncture is that we will need to know exactly when it might be the case that $x \in T \backslash C(T)$ and $x \succeq_{2} C(T)$ if we are to have any hope of proving that the construction method works.

It would be ideal to have a proposition of the form If $C$ is a two-tier choice function and $x \in T \backslash C(T)$, then $x \succeq_{2} C(T)$ if and only if ... We do not have this at our disposal, but we do have the following, starting with yet another definition:

Definition 11. $S_{T}(x, C(T))$ if and only if,

(1) $G_{T}\left(x, C\left(T^{\prime}\right)\right), S_{T}\left(C\left(T^{\prime}\right), y\right)$, and $G_{T}(y, C(T))$ [for some $\left.y, T^{\prime}\right]$, or

(2) for some $y_{1}, \ldots, y_{n}, T_{1}, \ldots, T_{n}$ :

(a) $G_{T}\left(x, C\left(T_{1}\right)\right)$, and

(b) $S_{T}\left(C\left(T_{i}\right), y_{i}\right)$ and $G_{T}\left(y_{i}, C\left(T_{i+1}\right)\right)$ [for all $i=1, \ldots, n-1$ ], and

(c) $S_{T}\left(C\left(T_{n}\right), y_{n}\right)$ and $G_{T}\left(y_{n}, C(T)\right)$.

The idea here is straightforward: if $C$ is a two-tier choice function and (1) holds then, by Lemmas 1-3 and the transitivity of $\succeq_{2}$, we have that $x \succeq_{2} C\left(T^{\prime}\right) \succ_{2} y \succeq_{2} C(T)$, or $x \succ_{2} C(T)$. Similarly for (2): if (2) holds and $C$ is a two-tier choice function then we have a chain of the following form:

$$
x \succeq_{2} C\left(T_{1}\right) \succ_{2} y_{1} \succeq_{2} C\left(T_{2}\right) \succ_{2} y_{2} \succeq_{2} \ldots \succeq_{2} C\left(T_{n}\right) \succ_{2} y_{n} \succeq_{2} C(T)
$$

And since $\succeq_{2}$ is transitive, this leaves us with $x \succ_{2} C(T)$.

In other words, the definition of $S_{T}(x, C(T))$ has been designed to capture certain situations where, if $C$ is a two-tier choice function, then $x \succ_{2} C(T)$. Further, the definition has been designed to capture all cases where the use of CR1-CR3 will produce an ordering $\succeq_{2}$ such that $x \succ_{2} C(T)$.

Lemma 6. If CR1-CR3 are used to construct $\succeq_{2}$ from $C$, then $x \succeq_{2} C(T)$ if and only if either $G_{T}(x, C(T))$ or $S_{T}(x, C(T))$. 
Proof. Suppose CR1-CR3 are used to construct $\succeq_{2}$ from a choice function, $C$. Let $x \in U$ and $T \subseteq U$.

We can think of the construction process of CR1-CR3 as a process of building chains of elements and choice sets, where CR3 ensures that the relation is transitive, and the appropriate preference relations hold for each pair of items along that chain. For instance, if $G_{T}(x, C(T))$ and $S_{T}(C(T), y)$ hold then, by CR1 and CR2, we have the chain $x \succeq_{2} C(T) \succ_{2} y$; and by CR3 it is also the case that $x \succ_{2} y$. In this chain, $x$ is the maximal item and $y$ is the minimal item.

Notice also that CR1 produces orderings of the form $x \succeq_{2} y-$ and $x \succeq_{2} C(T)$ if $I(\{y\} \cup C(T))$ holds - and CR2 produces orderings of the form $C(T) \succ_{2} x$. Here we are interested in situations where CR1-CR3 can be used to build the preference relation $x \succeq_{2} C(T)$, for some $x \in U$ and $T \subseteq U$. This will be done only if CR1 and CR2 combine to produce a chain where $x$ is the maximal item and $C(T)$ is the minimal item. Given the form of the orderings produced by CR1 and CR2, this can only occur if $G_{T}\left(x, C\left(T_{1}\right)\right)$ and $G_{T}\left(y_{n}, C(T)\right)$ hold for some set $T_{1}$ and element $y_{n}$. Now if $T_{1} \equiv T$ and/or $y_{n} \equiv x$ then we have a straightforward case: $x \succeq_{2} C(T)$ is built, by CR1, from $G_{T}(x, C(T))$.

But suppose $G_{T}(x, C(T))$ does not hold. Then, by CR1, we have $x \succeq_{2} C\left(T_{1}\right)$ and $y_{n} \succeq_{2} C(T)$, but we do not yet have the desired chain. What remains is to build a chain where the maximal item is $C\left(T_{1}\right)$ and the minimal item is $y_{n}$. Here it is relevant to notice the transitivity of $G_{T}$ : if $G_{T}\left(C\left(T_{1}\right), y_{n}\right)$ holds then so does $G_{T}(x, C(T))$. So, supposing that $G_{T}(x, C(T))$ does not hold, the desired chain will only result if $S_{T}\left(C\left(T_{1}\right), y_{1}\right)$ and $S_{T}\left(C\left(T_{n}\right), y_{n}\right)$ hold, for some element $y_{1}$ and set $T_{n}$. This, in combination with $G_{T}\left(x, C\left(T_{1}\right)\right)$ and $G_{T}\left(y_{n}, C(T)\right)$ gives us $x \succeq_{2} C\left(T_{1}\right) \succ_{2} y_{1}$ and $C\left(T_{n}\right) \succ_{2} y_{n} \succeq_{2} C(T)$. Of course, if $T_{1} \equiv T_{n}$ and/or $y_{1} \equiv y_{n}$ then we are done. Otherwise, to compete the chain and end up with the desired result that $x \succeq_{2} C(T)$, we must build a chain with $y_{1}$ as the maximal item and $C\left(T_{n}\right)$ as the minimal item. This effectively returns us to the beginning of the process: such a chain will require, since CR1-CR3 are the construction rules in use, that $G_{T}\left(y_{1}, C\left(T_{2}\right)\right)$ and $G_{T}\left(y_{n-1}, C\left(T_{n}\right)\right)$ hold for some set $T_{2}$ and element $y_{n-1}$. And again, if $T_{2} \equiv T_{n}$ and/or $y_{1} \equiv y_{n-1}$ then $G_{T}\left(y_{1}, C\left(T_{n}\right)\right)$ holds and we are done. Otherwise, $G_{T}\left(y_{1}, C\left(T_{n}\right)\right)$ does not hold, and we need to build a chain from $C\left(T_{2}\right)$ to $y_{n-1}$.

And we can continue iterating this procedure until a chain of the following form is built:

$$
x \succeq_{2} C\left(T_{1}\right) \succ_{2} y_{1} \succeq_{2} \ldots \succeq_{2} C\left(T_{n}\right) \succ_{2} y_{n} \succeq_{2} C(T)
$$

When this is built then, by CR3, we have that $x \succ_{2} C(T)$, as desired. And if such a 
chain cannot be built then it will never be the case that $x \succeq_{2} C(T)$.

With this in hand we can conclude that CR1-CR3 will conspire to produce $x \succeq_{2} C(T)$ [for some element $x$ and set $T$ ] in exactly three ways. First, if $G_{T}(x, C(T))$ holds then, by CR1, $x \succeq_{2} C(T)$. Second, if $G_{T}\left(x, C\left(T^{\prime}\right)\right), S_{T}\left(C\left(T^{\prime}\right), y\right)$ and $G_{T}(y, C(T))$ hold [for some $y, T^{\prime}$ ], then by CR1 and CR2 $x \succeq_{2} C\left(T^{\prime}\right) \succ_{2} y \succeq_{2} C(T)$; and by CR3 $x \succ_{2} C(T)$. And third, for some $y_{1}, \ldots, y_{n}$ and $T_{1}, \ldots, T_{n}[n \geq 2]$, if $G_{T}\left(x, C\left(T_{1}\right)\right)$ holds, $S_{T}\left(C\left(T_{i}\right), y_{i}\right)$ and $G_{T}\left(y_{i}, C\left(T_{i+1}\right)\right)$ hold for $i=1, \ldots, n-1$, and $S_{T}\left(C\left(T_{n}\right), y_{n}\right)$ and $G_{T}\left(y_{n}, C(T)\right)$ hold, then CR1-CR3 entail that $x \succ_{2} C(T)$. And the second and third cases are exactly those cases for which $S_{T}(x, C(T))$ holds.

So, if CR1-CR3 are used to construct the partial order $\succeq_{2}$ from $C$, then $x \succeq_{2} C(T)$ exactly when either $G_{T}(x, C(T))$ holds or $S_{T}(x, C(T))$ holds.

These feature will help our cause considerably. In order to proceed with the first-tier construction - in order to deal with TTP5 - we will need to know exactly when it is the case that $x \succeq_{2} C(T)$, if $x \in T \backslash C(T)$. To this end, our next construction rule ensures that, for any $x \in T \backslash C(T), C(T) \succ_{2} x$ unless CR1-CR3 have already established that $x \succeq_{2} C(T)$.

CR 4. $\forall x \in U, \forall T \subseteq U:$ If $x \in T \backslash C(T), \neg G_{T}(x, C(T))$, and $\neg S_{T}(x, C(T))$, then set $C(T) \succ_{2} x$.

In other words, if $x \in T \backslash C(T)$ and CR1-CR3 have not established that $x \succeq_{2} C(T)$, then set $C(T) \succ_{2} x$.

This fourth construction rule completes the framework for $V_{2}(U)$. We still need to ensure that the ordering is complete, but these first four rules are all that is required to set the stage. In addition, we know from Lemma 6 and the corresponding nature of CR4 that, for any $x \in T \backslash C(T), x \succeq_{2} C(T)$ if and only if either $G_{T}(x, C(T))$ or $S_{T}(x, C(T))$.

\subsection{First Tier Construction}

The first step in constructing $V_{1}(U)$ is to ensure that TTP4 is satisfied: to ensure that $C_{1}(S) \subseteq C(S)$ for all $S \subseteq U$. We can do this by constructing one first-tier ordering $\succeq_{S}$ for each $S \subseteq U$ that has at least two elements, ${ }^{10}$ and setting $C(S) \succeq_{S} S$. That is,

\footnotetext{
${ }^{10}$ The task is trivial for $|S|=1$ because $C(\{x\})=C_{1}(\{x\})=C_{2}\left(C_{1}(\{x\})\right)=\{x\}$, provided the orderings are complete.
} 
CR 5. For each $S \subseteq U$ where $|S|>1$, construct $\succeq_{S}$ such that: For any $x, y \in U$ where $x \in C(S)$ and $y \in S$, set $x \succeq_{S} y$.

The second step in constructing $V_{1}(U)$ is far more complicated. Recall our fifth twotier property:

TTP 5. $\forall T \subseteq U$ : If $x \in T \backslash C(T)$ and $x \succeq_{2} C(T)$, then $x \notin C_{1}(T)$.

From above we know that $x \in T \backslash C(T)$ and $x \succeq_{2} C(T)$ (when CR1-CR4 are used) if and only if $G_{T}(x, C(T))$ or $S_{T}(x, C(T))$. So the general idea for the next construction rule is this:

CR 6. (general) For each $\succeq_{S} \in V_{1}(U): \forall x \in U, \forall T \subseteq U$ such that $x \in T \backslash C(T)$ and either $G_{T}(x, C(T))$ or $S_{T}(x, C(T))$, pick some appropriate $y \in T \backslash\{x\}$ and set $y \succ_{S} x$.

To move from this general version to a more helpful construction rule we first need to unpack this notion of 'appropriateness'. Most straightforwardly, an element $y \in$ $T \backslash\{x\}$ is 'appropriate' if setting $y \succ_{S} x$ will not produce any cycles in $\succeq_{S}$, given the rest of the construction procedure.

For example, if $x \in C(S)$ then $x \succeq_{S} S$; and so, if $x \in T \backslash C(T)$ and $x \succeq_{2} C(T)$ we will need a $y \in T \backslash S$. Further, suppose we set $y \succ_{S} x$ but it also turns out that $y \in T^{\prime} \backslash C\left(T^{\prime}\right)$ and $y \succeq_{2} C\left(T^{\prime}\right)$ [for some $T^{\prime} \subseteq U$ ]. Then we need some $z \in T^{\prime} \backslash(S \cup\{y\})$ if we are to set $z \succ_{S} y \succ_{S} x$. And if $z \in T^{\prime \prime} \backslash C\left(T^{\prime \prime}\right)$ and $z \succeq_{2} C\left(T^{\prime \prime}\right)$ then we need some appropriate $w$. And so the pattern continues.

But before we get too complicated, a simpler example is in order.

Example 2. Let $U=\{a, b, c\}$ and define $C$ such that $C(\{a, b, c\})=C(\{a, c\})=$ $\{a, c\}$ and $C(\{a, b\})=C(\{b, c\})=\{b\}$.

Notice first that $I(\{a, c\})$ holds. So $G_{T}(a, c)$ and $G_{T}(c, a)$ hold: by CR1 we set $a \succeq_{2} c \succeq_{2} a$, or $a \sim_{2} c$. Further, $b \in\{a, b, c\}$ and $C(\{a, b\})=C(\{b, c\})=\{b\}$, so $G_{T}(b, a)$ and $G_{T}(b, c)$ hold: by CR1 we set $b \succeq_{2} a$ and $b \succeq_{2} c$. And finally, $S_{T}(C(\{a, b\}), a)$ and $S_{T}(C(\{b, c\}), c)$ hold - or $a \in C(\{a, b, c\}) \cap\{a, b\} \backslash C(\{a, b\})$ and $c \in C(\{a, b, c\}) \cap\{b, c\} \backslash C(\{b, c\})$. So, by CR2, $b \succ_{2} a$ and $b \succ_{2} c$. In other words, CR1 and CR2 give us the transitive second-tier ordering $b \succ_{2} a \sim_{2} c$. In this case, CR3 and CR4 do not add anything to the construction.

For the first tier construction let $S_{1}=\{a, b, c\}, S_{2}=\{a, b\}, S_{3}=\{a, c\}$, and $S_{4}=\{b, c\}$. By CR5, then, we will construct four first-tier orderings. Based on the definition of $C$, our starting point for each ordering is 


$$
\begin{gathered}
a \sim_{S_{1}} c ; a, c \succeq_{S_{1}} b \\
b \succeq_{S_{2}} a \\
a \sim_{S_{3}} c \\
b \succeq_{S_{4}} c
\end{gathered}
$$

To apply CR6 it is helpful to first identify the $\langle x, T>$ pairs to which it applies. In this case there is only one such $\langle x, T>$ pair:

$$
b \in\{a, b, c\} \backslash C(\{a, b, c\}) \text { but } G_{T}(b, C(\{a, b, c\}))
$$

So for each $\succeq_{S} \in V_{1}(U)$ we must set either $a \succ_{S} b$ or $c \succ_{S} b$.

Recall that $S_{1}=\{a, b, c\}, a \sim_{S_{1}} c$ and $a, c \succeq_{S_{1}} b$. To satisfy CR6 our only option is to strengthen the weak preference to strict: $\succeq_{S_{1}}$ becomes $a \sim_{S_{1}} c \succ_{S_{1}} b$. Similarly, for $S_{3}=\{a, c\}$ our only option is to set $a \sim_{S_{3}} c \succ_{S_{3}} b .{ }^{11}$

For $S_{2}=\{a, b\}$ we know that $b \succeq_{S_{2}} a$. So if we set $a \succ_{S_{2}} b$ then we will generate a cycle. The element $a$ is not an 'appropriate' choice. Rather, we set $c \succ_{S_{2}} b \succeq_{S_{2}} a$. Similarly for $S_{4}=\{b, c\}$ : we set $a \succ_{S_{4}} b \sim_{S_{4}} c$.

So now we have constructed $V_{1}(U)$ and $V_{2}(U)$ for the given choice function:

$$
\begin{gathered}
a \sim_{S_{1}, S_{3}} c \succ_{S_{1}, S_{3}} b \\
c \succ_{S_{2}} b \succeq_{S_{2}} a \\
a \succ_{S_{4}} b \succeq_{S_{4}} c \\
b \succ_{2} a \sim_{2} c
\end{gathered}
$$

A quick look verifies that, based on the constructed orderings, $C_{2}\left(C_{1}(S)\right)=C(S)$ for all $S \subseteq U$. This does not give us a proof of anything, but it should provide some hope that we are on the right track.

Unfortunately, far more complicated examples abound and CR6 must be further specified to deal with increasingly complex examples. This specification of CR6 is rather tedious, but unless the procedure is laid out with particulars we cannot show that our construction rules can be used to build $V_{1}(U)$ and $V_{2}(U)$ without producing cycles. It is to this task that I now turn my attention.

\footnotetext{
${ }^{11}$ In fact we set either $a \succ_{S} b$ or $c \succ_{S} b$. The ordering here assumes transitivity. I have been a little lax in this example by assuming transitivity, but this will be included explicitly as Construction Rule 7.
} 


\subsubsection{Specifying CR6}

To get an idea of the contents of our further specified CR6, first notice that if $x \in$ $T \backslash C(T)$ [for some $T \subseteq U$ ] then there is some $y \in T \backslash\{x\}$ : because $C(T) \neq \emptyset$ and $x \notin C(T)$. Without loss of generality, suppose we are building the first-tier ordering $\succeq_{S}$, for some arbitrary $S \subseteq U$, and suppose $x_{1} \in T_{1} \backslash C\left(T_{1}\right)$ and $x_{1} \succeq_{2} C\left(T_{1}\right)$. We will select some $x_{2} \in T_{1} \backslash\left\{x_{1}\right\}$ and set $x_{2} \succ_{S} x_{1}$.

Suppose further that $x_{2} \in T_{2} \backslash C\left(T_{2}\right)$ and $x_{2} \succeq_{2} C\left(T_{2}\right)$ [for some $T_{2} \subseteq U$ ]. If there is an $x_{3} \in T_{2} \backslash\left\{x_{1}, x_{2}\right\}$ then we can straightforwardly set $x_{3} \succ_{S} x_{2} \succ_{S} x_{1}$. If there is no such element then we will need to select $x_{3} \in T_{2} \backslash\left\{x_{2}\right\}$ and $x_{3}^{\prime} \in T_{1} \backslash\left\{x_{1}\right\}$, and set $x_{3} \succ_{S} x_{2}$ and $x_{3}^{\prime} \succ_{S} x_{1}$. It might turn out that $x_{3}=x_{1}$, but this detail is unimportant. The point here is just to sketch a procedure for an application of CR6.

Of course, if $x_{3} \in T_{3} \backslash C\left(T_{3}\right)$ and $x_{3} \succeq_{2} C\left(T_{3}\right)$ [for some $T_{3} \subseteq U$ ] then we will need to find $x_{4} \in\left(T_{1} \cup T_{2} \cup T_{3}\right) \backslash\left\{x_{1}, x_{2}, x_{3}\right\}$ and re-set the order accordingly.

What follows is a preliminary specification of CR6. More detail needs to be added to account for situations where $x \in C(S) \cap T \backslash C(T)$ and $x \succeq_{2} C(T)$ [for some $S, T \subseteq U$ ], but these details will be easier to handle after we have a version of CR6 in hand, and will only make our rule more tedious if we include them from the outset.

The specification of CR6 is presented next, followed immediately with an explanation of its contents.

CR 6. (specific) For each $\succeq_{S} \in V_{1}(U)$ :

Let $\chi^{\prime}=\left\{\langle x, T, Y\rangle \mid T \subseteq U, x \in T \backslash C(T), x \succeq_{2} C(T)\right.$, and $Y=$ $\{y \mid y \in T \backslash\{x\}$ and, if $x \in C(S)$, then $y \notin S\}\}$.

Let $\chi=\left\{\langle x, T, Y\rangle|<x, T, Y\rangle \in \chi^{\prime}\right.$ and $\neg \exists T^{\prime} \subseteq T$ s.t. $<$ $\left.x, T^{\prime}, Y^{\prime}>\in \chi^{\prime}\right\}$.

\section{Step 1:}

Start with an arbitrary $<x_{1}, T_{1}, Y>\in \chi$.

If $\exists x_{2} \in Y_{1}$ where $\neg \exists T_{2}, Y_{2}$ s.t. $<x_{2}, T_{2}, Y_{2}>\in \chi$, then set $x_{2} \succ_{S} x_{1}$ and proceed to Step $1^{\prime}$.

Otherwise, pick $x_{2} \in Y_{1}$, set $x_{2} \succ_{S} x_{1}$, and proceed to Step 2 .

Step n: $[n \geq 2]$

A chain of the following form, with $<x_{n}, T_{n}, Y_{n}>\in \chi$, has been established: 


$$
x_{n} \succ_{S} x_{n-1} \succ_{S} \ldots \succ_{S} x_{2} \succ_{S} x_{1}
$$

If $\exists x_{n+1} \in Y_{n} \backslash\left\{x_{1}, \ldots, x_{n}\right\}$ where $\neg \exists T_{n+1}, Y_{n+1}$ such that $<$ $x_{n+1}, T_{n+1}, Y_{n+1}>\in \chi$, then set $x_{n+1} \succ_{S} x_{n}$ and proceed to Step $1^{\prime}$.

Otherwise,

(1) If $\exists x_{n+1} \in Y_{n} \backslash\left\{x_{1}, \ldots, x_{n}\right\}$ then set that $x_{n+1} \succ_{S} x_{n}$ and proceed to Step $(n+1)$. Otherwise, proceed to $(2)$. $\vdots$

(i) If $\neg \exists x_{n+1} \in Y_{n-j} \backslash\left\{x_{1}, \ldots, x_{n}\right\}$ [for all $j=0, \ldots, i-2$ ] but $\exists x_{n+1} \in Y_{n-(i-1)} \backslash\left\{x_{1}, \ldots, x_{n}\right\}$, then pick that $x_{n+1}$ and set $x_{n+1} \succ_{S} x_{n-(i-1)} \succ_{S} x_{n-(i-2)} \succ_{S} \ldots \succ_{S} x_{2} \succ_{S} x_{1} \succ_{S} x_{n} \succ_{S}$ $x_{n-1} \succ_{S} \ldots \succ_{S} x_{n-i}$ and proceed to Step $(n+1)$. Otherwise, proceed to $(i+1)$.

\section{Step 1':}

At least one chain of the following form has been established:

$$
x_{m} \succ_{S} x_{m-1} \succ_{S} \ldots \succ_{S} x_{2} \succ_{S} x_{1}
$$

For all $i=1, \ldots, m-1$ we have $<x_{i}, T_{i}, Y_{i}>\in \chi$. And $\neg \exists T_{m}, Y_{m}$ s.t. $<x_{m}, T_{m}, Y_{m}>\in \chi$.

Pick $<y_{1}, S_{1}, Z_{1}>\in \chi$ such that $<y_{1}, S_{1}, Z_{1}>\neq<x_{i}, T_{i}, Y_{i}>$ for any $i=1, \ldots, m-1$. If no such triplet exists, repeat the entire process for the next $\succeq_{S} \in V_{1}(U)$ [or proceed to CR7, if no $\succeq_{S} \in V_{1}(U)$ remains].

If $y_{1}=x_{k}$ for some $k=1, \ldots, m-1$ then we already have a chain of the form $y_{1} \equiv x_{k} \succ_{S} x_{k-1} \succ_{S} \ldots \succ_{S} x_{2} \succ_{S} x_{1}$. Proceed to Step $\mathrm{k}$ and continue accordingly.

Otherwise, follow the pattern of Step 1: pick $y_{2} \in Z_{1} \backslash\left\{y_{1}\right\}$, set $y_{2} \succ_{S} y_{1}$, and proceed to either Step 2 or Step $1^{\prime}$.

\subsubsection{An Explanation}

To understand the rationale behind this more specified version of CR6, a few comments are in order. First, notice that $Y$ is just the set of elements that might be used to prevent $x$ from first-tier admissibility in $T$. If $x \in C(S)$ then this set cannot 
include any $y \in S$. Second, consider the reduction of $\chi^{\prime}$ to $\chi$. Let $<x, T, Y>\in \chi$ and $<x, T^{\prime}, Y^{\prime}>\in \chi^{\prime}$, with $T \subseteq T^{\prime}$. Suppose we pick some $y \in T \backslash\{x\}$ and set $y \succ_{S} x$ so that $x \notin C_{1}(T)$. $C_{1}$ satisfies Property $\alpha$, so this also means that $x \notin C_{1}\left(T^{\prime}\right)$. Proceeding through the algorithm for the set $\chi$ instead of $\chi^{\prime}$ eliminates some redundancy.

In Step 1, we start with an arbitrary $x_{1} \in T_{1} \backslash C\left(T_{1}\right)$ and $x_{1} \succeq_{2} C\left(T_{1}\right) . Y_{1}$ is the set of elements that we might use to prevent $x_{1}$ from first-tier admissibility in $T_{1}$. Ideally, there is some $x_{2} \in Y_{1}$ that we can use for this purpose, while not also having $x_{2} \in T_{2} \backslash C\left(T_{2}\right)$ and $x_{2} \succeq_{2} C\left(T_{2}\right)$. If so, we can set $x_{2} \succ_{S} x_{1}$ and we have successfully prevented $x_{1}$ from first-tier admissibility in $C\left(T_{1}\right)$ (for this ordering, at least); and we can turn our attention to some other $\langle x, T, Y\rangle$ triple in $\chi$. If no such $x_{2}$ exists, then we are forced to construct a longer chain. We can pick $x_{2} \in Y_{1}$ and set $x_{2} \succ_{S} x_{1}$, but we still need to prevent $x_{2}$ from first-tier admissibility in $T_{2}$ : so we proceed to Step 2.

For Step 2 we follow the algorithm laid out by Step $\mathrm{n}$, for $n=2$. Once again, it is most expedient to set $x_{3} \succ_{S} x_{2} \succ_{S} x_{1}$ and solve all of our problems. But if no appropriate $x_{3}$ exists, then we must turn to a slightly more complicated algorithm.

If there is some $x_{3} \in Y_{2} \backslash\left\{x_{1}, x_{2}\right\}$, then we can straightforwardly set $x_{3} \succ_{S} x_{2} \succ_{S} x_{1}$. Since $x_{3}$ has its own problems, we proceed to Step 3 and continue. If there is no such $x_{3}$ then we proceed to (2) - which is laid out by (i), with $i=2$. There is no $x_{3} \in Y_{2} \backslash\left\{x_{1}, x_{2}\right\}$, but perhaps there is some $x_{3} \in Y_{1} \backslash\left\{x_{1}, x_{2}\right\}$. If so, then we can use that $x_{3}$ and set $x_{3} \succ_{S} x_{1} \succ_{S} x_{2}$. This blocks $x_{1}$ from admissibility in $C_{1}\left(T_{1}\right)$, as desired. Also, since we are currently only considering situations where $x_{1}, x_{2}, x_{3} \notin C(S)$, we know that $x_{1} \in T_{2} \backslash\left\{x_{2}\right\}$. That is, $Y_{2} \neq \emptyset, x_{2} \notin Y_{2}$, and there is no $x_{3} \in Y_{2} \backslash\left\{x_{1}, x_{2}\right\}$; so $x_{1} \in Y_{2}$. And hence setting $x_{1} \succ_{S} x_{2}$ successfully blocks $x_{2}$ from first-tier admissibility. We are free to proceed to Step 3, which follows a pattern that is identical to Step 2. And this pattern continues until we have an ordering of the form identified in Step $1^{\prime}$.

Now consider what is being built. We begin with some $\langle x, T, Y\rangle \in \chi$ and we proceed through a series of steps until we reach Step 1'. At this point we will have defined $\succeq_{S}$ such that $C(S) \succeq_{S} S$, and there is some chain of the form $x_{m} \succ_{S} \ldots \succ_{S} x_{1}$. The spirit of Step $1^{\prime}$ is to pick some $\langle x, T, Y\rangle$ triplet in $\chi$ for which it might still be the case that $x \in C_{1}(T) . x_{1}$ through $x_{m}$ are no longer a worry, so we turn our attention elsewhere.

Suppose we arrive at Step $1^{\prime}$ and we pick $<y_{1}, S_{1}, Z_{1}>\in \chi$. One of four things might happen as a result of this choice:

\section{Scenario 1: New Chain}


If $y_{1} \notin\left\{x_{1}, \ldots, x_{m-1}\right\}$ then we pick $y_{2} \in Z_{1} \backslash\left\{y_{1}\right\}$, set $y_{2} \succ_{S} y_{1}$, and proceed with Step 2 (or Step 1', if $y_{2}$ is problem-free). Upon return to Step $1^{\prime}$ we will have two chains:

$$
\begin{gathered}
x_{m} \succ_{S} \ldots \succ_{S} x_{1} \\
\quad \text { and } \\
y_{p} \succ_{S} \ldots \succ_{S} y_{1} .
\end{gathered}
$$

\section{Scenario 2: Bottom Branching}

In this case we start out just like Scenario 1, but we end up with some $y_{p} \in\left\{x_{2}, \ldots, x_{m}\right\}$. Upon return to Step $1^{\prime}$ we have two chains that share a common part:

$$
\begin{gathered}
x_{m} \succ_{S} \ldots \succ_{S} x_{1} \\
\text { and } \\
x_{m} \succ_{S} \ldots \succ_{S} x_{k} \equiv y_{p} \succ_{S} \ldots \succ_{S} y_{1}
\end{gathered}
$$

\section{Scenario 3: Extended Chain}

This case is identical to Scenario 2 except that we end up with $y_{p}=x_{1}$ or $x_{1} \succ_{S} y_{p}$ The result is a single, extended chain:

$$
\begin{gathered}
x_{m} \succ_{S} \ldots \succ_{S} x_{1} \equiv y_{p} \succ_{S} \ldots \succ_{S} y_{1} \\
\text { or } \\
x_{m} \succ_{S} \ldots \succ_{S} x_{1} \succ_{S} y_{p} \succ_{S} \ldots \succ_{S} y_{1}
\end{gathered}
$$

\section{Scenario 4: Top Branching}

Suppose $y_{1} \in\left\{x_{2}, \ldots, x_{m-1}\right\}$. Then we have $y_{1} \equiv x_{k} \succ_{S} \ldots \succ_{S} x_{1}$. We can proceed to Step k, picking $y \in T \backslash\{x\}$. Upon return to Step $1^{\prime}$ we have two chains that share a common part:

$$
\begin{gathered}
x_{m} \succ_{S} x_{m-1} \succ_{S} \ldots \succ_{S} x_{2} \succ_{S} x_{1} \\
\text { and } \\
y_{p} \succ_{S} \ldots \succ_{S} y_{1} \equiv x_{k} \succ_{S} \ldots \succ_{S} x_{1}
\end{gathered}
$$


Whichever scenario obtains, Step 1' should be altered accordingly. In most cases, we end up with two chains after the second pass, not one. For the next $\langle x, T, Y\rangle$ we choose, we can consider its relation to either chain. If $x=x_{k}$ for $k=1, \ldots m-1$ or $x=y_{k}$ for $k=1, \ldots, p$, then a branching chain is in order. Otherwise, we build the new chain up until it meets one of the previously established chains, or until we can return to Step 1'.

And our arrival at Step $1^{\prime}$ now includes one, two, or three chains.

\subsubsection{What does CR6 Require?}

All of this, of course, relies on the existence of the appropriate elements at each step along the way. For Step 1, it need only be the case that $Y_{1} \neq \emptyset$. If $x_{1} \in T_{1} \backslash C\left(T_{1}\right)$ and $x_{1} \succeq_{2} C\left(T_{1}\right)$ then there must be some $x_{2} \in T_{1} \backslash\left\{x_{1}\right\}$.

In general, CR6 produces strict orderings of elements: chains of the form $x_{n} \succ_{S}$ $x_{n-1} \succ_{S} \ldots \succ_{S} x_{2} \succ_{S} x_{1}$. At each step along the way, if $x_{n} \in T_{n} \backslash C\left(T_{n}\right)$ and $x_{n} \succeq_{2} C\left(T_{n}\right)$ [for some $T_{n} \subseteq U$ ], then we have more work to do. In the most basic case, $x_{i} \notin C(S)$ for any $i=1, \ldots, n$.

To successfully complete Step n of CR6 there must be some $x_{n+1} \in\left(T_{1} \cup \ldots \cup T_{n}\right) \backslash$ $\left\{x_{1}, \ldots, x_{n}\right\}$, and it must not be the case that $Y_{i}=\emptyset$ for any $i$. That is to say, the following condition must be satisfied if CR6 is to be successfully applied:

\section{Proposed Condition 2. TTIC(1)}

(a) For any $x \in U, T \subseteq U$ : If $x \in T \backslash C(T)$ then $\exists y \in C(T) \backslash\{x\}$.

(b) For any sequence of sets $T_{1}, \ldots, T_{n} \subseteq U$, if there is a corresponding sequence of elements $x_{1}, \ldots, x_{n} \in U[n \geq 2]$ such that:

$$
\begin{aligned}
& x_{1} \in T_{1} \backslash C\left(T_{1}\right), \text { and } \\
& x_{i} \in\left(\left(T_{1} \cup \ldots \cup T_{i-1}\right) \cap T_{i}\right) \backslash\left(C\left(T_{i}\right) \cup\left\{x_{1}, \ldots, x_{i-1}\right\}\right)[\text { for } i=2, \ldots, n], \\
& \text { and } \\
& G_{T}\left(x_{i}, C\left(T_{i}\right)\right) \text { or } S_{T}\left(x_{i}, C\left(T_{i}\right)\right)[\text { for } i=1, \ldots, n]
\end{aligned}
$$$$
\text { then } \exists y \in\left(T_{1} \cup \ldots \cup T_{n}\right) \backslash\left\{x_{1}, \ldots, x_{n}\right\} \text {. }
$$

Recall from Lemma 6 (and the related nature of CR4) that when CR1-CR4 are used to construct $\succeq_{2}, x \succeq_{2} C(T)$ if and only if $G_{T}(x, C(T))$ or $S_{T}(x, C(T))$. So the antecedent of TTIC(1) lays out a scenario where $x_{i} \in T_{i} \backslash C\left(T_{i}\right)$ and $x_{i} \succeq_{2} C\left(T_{i}\right)$, for each $i=1, \ldots, n$ (when CR1-CR4 are used to construct $\succeq_{2}$ ). 
Further, any chain of the form $x_{n+1} \succ_{S} x_{n} \succ_{S} \ldots \succ_{S} x_{2} \succ_{S} x_{1}$ produced by CR6 (in conjunction with CR1-CR4) will consist of elements $x_{1}, \ldots, x_{n}$ that satisfy the antecedent of TTIC(1). ${ }^{12}$ And, by (a), we know that $Y_{i} \neq \emptyset$ for any $i$.

So if TTIC(1) is satisfied by a choice function $C$, then CR6 can be successfully completed - in the most basic cases where $x_{i} \notin C(S)$.

\subsubsection{What if $x \in C(S)$ ?}

But suppose $x_{n} \succ_{S} \ldots \succ_{S} x_{1}$ and $x_{1} \in C(S)$. Then the antecedent of TTIC(1) holds, but it also must be true that $x_{i} \notin S$, for each $i=2, \ldots, n$. It is to this additional feature that I now turn my attention.

The main thing to note is that if $x \in C(S)$ then, by CR5, $x \succeq_{S} S$. So whichever element we choose to use to block $x$ from first-tier admissibility in $T$ (where $x \in$ $T \backslash C(T)$ and $\left.x \succeq_{2} C(T)\right)$, it cannot be an element of $S$. That is, if $x \in C(S) \cap T \backslash C(T)$ and $x \succeq_{2} C(T)$ then we need to select $y \in T \backslash S$ and set $y \succ_{S} x \succeq_{S} S$.

All things considered, the use of CR6 might bring us to one of two relevant situations: (1) We pick $\langle x, T, Y\rangle$ at Step 1 or Step $1^{\prime}$, and $x \in C(S)$, or (2) We build a chain of the form $x_{n} \succ_{S} \ldots \succ_{S} x_{1},<x_{n}, T_{n}, Y_{n}>\in \chi$, and $x_{n} \in C(S)$.

In the first case we must select $x_{2} \in T_{1} \backslash S$ and set $x_{2} \succ_{S} x_{1} \succeq_{S} S$. If $<x_{2}, T_{2}, Y_{2}>\in \chi$ then we proceed to Step 2. If there is some $x_{3} \in T_{2} \backslash\left(S \cup\left\{x_{1}, x_{2}\right\}\right)$ then we can use that $x_{3}$ and set $x_{3} \succ_{S} x_{2} \succ_{S} x_{1} \succeq_{S} S$ and move on to Step 3 (or Step 1'), following the same pattern. If no such $x_{3}$ exists then we know that $T_{2} \backslash\left\{x_{2}\right\} \subseteq S$. So we instead pick $x_{3} \in T_{1} \backslash\left(S \cup\left\{x_{1}, x_{2}\right\}\right)$ and $x_{3}^{\prime} \in T_{2} \backslash\left\{x_{2}\right\}$, and set $x_{3} \succ_{S} x_{1} \succeq_{S} x_{3}^{\prime} \succ_{S} x_{1}$ (with $x_{1} \succeq_{S} S$ and $\left.x_{3}^{\prime} \in S\right)$.

This effectively gives us two chains to deal with: $x_{3} \succ_{S} x_{1} \succeq_{S} x_{3}^{\prime} \succ_{S} x_{2}$, and $x_{3}^{\prime} \succ_{S} x_{1}$. The second one is straightforward, and we can deal with it at Step 2 of CR6, as specified above (if needed). For the first one, if $x_{3} \in T_{3} \backslash C\left(T_{3}\right)$ and $x_{3} \succeq_{2} C\left(T_{3}\right)$ then we need some $x_{4} \in T_{3} \backslash\left(S \cup\left\{x_{1}, x_{2}\right\}\right)$. If some such $x_{4}$ exists then we set $x_{4} \succ_{S} x_{3}$ and continue. If not, then we again split the ordering: pick $x_{4} \in T_{1} \backslash\left(S \cup\left\{x_{2}, x_{3}\right\}\right)$ and $x_{4}^{\prime} \in T_{3} \backslash\left\{x_{3}\right\}$, and set $x_{4} \succ_{S} x_{1} \succeq_{S} x_{4}^{\prime} \succ_{S} x_{3}$ (with $x_{3}^{\prime} \succ_{S} x_{2}, x_{1} \succeq_{S} S$, and $\left.x_{4}^{\prime} \in S\right)$. And again we have two chains to deal with, and we can continue in the manner just described.

Of course, all of this requires, once again, the existence of certain types of elements. If we build a chain of the form $x_{n} \succ_{S} \ldots \succ_{S} x_{1} \succeq_{S} S$, with $x_{n} \in T_{n} \backslash C\left(T_{n}\right)$ and

\footnotetext{
${ }^{12}$ That is, if $x_{n+1} \succ_{S} x_{n} \succ_{S} \ldots \succ_{S} x_{2} \succ_{S} x_{1}$, by CR6, then there is some sequence of sets $T_{1}, \ldots, T_{n} \subseteq U$ such that the antecedent of TTIC(1) holds.
} 
$x_{n} \succeq_{2} C\left(T_{n}\right)$ then there must be some $y \in\left(T_{1} \cup \ldots \cup T_{n}\right) \backslash\left(S \cup\left\{x_{1}, \ldots, x_{n}\right\}\right)$ - if we are to succeed in preventing each $x_{i}$ from first-tier admissibility in $T_{i}$ (without producing cycles in our ordering). The following condition guarantees that just such an element will be available.

\section{Proposed Condition 3. TTIC(2)}

(a) For any $S, T \subseteq U$ : if $x \in C(S) \cap T \backslash C(T)$ and either $G_{T}(x, C(T))$ or $S_{T}(x, C(T))$, then $\exists y \in T \backslash S$.

(b) For any sequence of sets $S, T_{1}, \ldots, T_{n} \subseteq U[n \geq 2]$, if there is a corresponding sequence of elements $x_{1}, \ldots, x_{n}$ such that

$$
\begin{aligned}
& x_{1} \in C(S) \cap T_{1} \backslash C\left(T_{1}\right), \text { and } \\
& x_{i} \in\left(\left(T_{1} \cup \ldots \cup T_{i-1}\right) \cap T_{i}\right) \backslash\left(C\left(T_{i}\right) \cup S \cup\left\{x_{1}, \ldots, x_{i-1}\right\}\right)[i=2, \ldots, n], \\
& \text { and } \\
& G_{T}\left(x_{i}, C\left(T_{i}\right)\right) \text { or } S_{T}\left(x_{i}, C\left(T_{i}\right)\right)[\mathrm{i}=1, \ldots, \mathrm{n}]
\end{aligned}
$$

then $\exists y \in\left(T_{1} \cup \ldots \cup T_{n}\right) \backslash\left(S \cup\left\{x_{2}, \ldots, x_{n}\right\}\right)$.

In the chain under consideration, $x_{1}, \ldots, x_{n}$ meet the conditions set out in the antecedent, and hence we can conclude, with $\operatorname{TTIC}(2)$, that there is some $y \in\left(T_{1} \cup \ldots \cup\right.$ $\left.T_{n}\right) \backslash\left(S \cup\left\{x_{1}, \ldots, x_{n}\right\}\right)$. CR6 can be successfully applied.

\subsubsection{Some Remaining Issues}

A few issues remain before we can declare our discussion of CR6 complete. For instance, suppose we begin with $x_{1} \in C(S) \cap T_{1} \backslash C\left(T_{1}\right)$ and $x_{1} \succeq_{2} C\left(T_{1}\right)$, proceeding as described above, and we build a chain of the form $x_{n} \succ_{S} \ldots \succ_{S} x_{j+1} \succeq_{S} x_{j}^{\prime} \succ_{S}$ $x_{j} \succ_{S} \ldots \succ_{S} x_{1}$ (with $x_{j+1} \succeq_{S} S$ ). What guarantee is there that, if needed, we can reconfigure the ordering such that $x_{n}$ is not first-tier admissible in $T_{n}$ ? Or suppose we end up in a 'bottom branching' scenario - where $x_{n} \succ_{S} \ldots \succ_{S} x_{1}$ and $x_{j} \succ_{S} y_{m} \succ_{S}$ $\ldots \succ_{S} y_{1}$ (for some $j=2, \ldots m$ ). What guarantee is there that the ordering can be configured in such a way that cycles are avoided?

I will not belabour the explanation and proof here, but it turns out that TTIC(1) and TTIC(2) are all that is needed to ensure that CR6 can be successfully completed without producing untoward cycles in the constructed ordering $\succeq_{S}$. A full proof requires spelling out CR6 in yet more detail, but hopefully you are beginning to see the pattern - and the power that the existence claims in TTIC(1) and TTIC(2) bring 
with them. Provided a choice function, $C$, satisfies TTIC(1) and TTIC(2), CR6 can be used, alongside CR1-CR5, to produce well-formed partial orderings in $V_{1}(U)$ - one ordering for each $S \subseteq U$ that has at least two elements.

\subsection{Completing the Orderings}

At this point we have six construction rules in play. When used together these rules will produce a single partial ordering in the second tier, and multiple partial orderings in the first tier.

CR 1. $\forall<x, y>\in U^{2}$ : If $G_{T}(x, y)$ then set $x \succeq_{2} y$.

CR 2. $\forall x \in U, \forall T \subseteq U$ : If $S_{T}(C(T), x)$ then set $C(T) \succ_{2} x$.

CR 3. $\forall x, y, z \in U$ :

If $x \succeq_{2} y$ and $y \succeq_{2} z$ then set $x \succeq_{2} z$.

If $x \succ_{2} y$ and $y \succeq_{2} z$ then set $x \succ_{2} z$.

If $x \succeq_{2} y$ and $y \succ_{2} z$ then set $x \succ_{2} z$.

CR 4. $\forall x \in U, \forall T \subseteq U$ : If $x \in T \backslash C(T), \neg G_{T}(x, C(T))$, and $\neg S_{T}(x, C(T))$, then set $C(T) \succ_{2} x$.

CR 5. For each $S \subseteq U$ where $|S|>1$, construct $\succeq_{S}$ such that: For any $x, y \in U$ where $x \in C(S)$ and $y \in S$, set $x \succeq_{S} y$.

CR 6. (general) For each $\succeq_{S} \in V_{1}(U): \forall x \in U, \forall T \subseteq U$ such that $x \in T \backslash C(T)$ and $G_{T}(x, C(T))$ or $S_{T}(x, C(T))$, pick some appropriate $y \in T \backslash\{x\}$ and set $y \succ_{S} x$.

Part of the second tier ordering is already transitive, and while my discussion of CR6 proceeded as though the ordering was transitive, this feature needs to be explicitly established.

CR 7. $\forall \succeq_{i} \in V_{1}(U) \cup V_{2}(U): \forall x, y, z \in U$

$$
\begin{aligned}
& \text { If } x \succeq_{i} y \text { and } y \succeq_{i} z \text { then set } x \succeq_{i} z . \\
& \text { If } x \succ_{i} y \text { and } y \succeq_{i} z \text { then set } x \succ_{i} z . \\
& \text { If } x \succeq_{i} y \text { and } y \succ_{i} z \text { then set } x \succ_{i} z .
\end{aligned}
$$

Provided CR6 has been applied with an eye to the details, CR7 will not produce any cycles in the orderings. So now we have transitive partial orderings; we need to complete the orderings before we can declare the construction process finis. 
CR 8. For each $\succeq_{i} \in V_{1}(U) \cup V_{2}(U)$, complete $\succeq_{i}$ as follows:

(a) Define $\mathbb{C}:=\left\{\langle x, y\rangle \mid x, y \in U, \neg\left(x \succeq_{i} y\right)\right.$, and $\left.\neg\left(y \succeq_{i} x\right)\right\}$.

(b) If $\mathbb{C}=\emptyset$ then the process is complete for $\succeq_{i}$. Otherwise, pick some $<x, y>\in \mathbb{C}$ and set $x \sim_{i} y$.

(c) Apply CR7 to $\succeq_{i}$ (to maintain transitivity).

(d) Return to (a), re-defining $\mathbb{C}$.

If no relationship has been established by CR1-CR7 between two elements, $x$ and $y$, for some ordering $\succeq_{i}$, then it does not matter what that relationship is. CR1-CR7 do the bulk of the work that is needed to ensure that $C(\cdot)=C_{2}\left(C_{1}(\cdot)\right)$. CR8 simply completes the picture to ensure that $C_{2}\left(C_{1}(S)\right)$ is defined for all $S \subseteq U$.

\subsection{The Success of CR1-CR8}

Of course, it remains to be shown that CR1-CR8 do, in fact, ensure that $C(\cdot)=$ $C_{2}\left(C_{1}(\cdot)\right)$. Without such a proof, we cannot say that CR1-CR8 are sufficient as construction rules. It is to this issue that I now turn my attention.

Proposition 1. If $C R 1-C R 8$ are used to construct acyclic orderings in $V_{1}(U)$ and $V_{2}(U)$, then $x \in C(T)$ if and only if $x \in C_{2}\left(C_{1}(T)\right)$ [for all $T \subseteq U$ ].

Proof. Suppose CR1-CR8 are used to construct acyclic orderings in $V_{1}(U)$ and $V_{2}(U)$. In the forward direction, let $a \in C(T)$ for some $T \subseteq U$. Then, by CR5, $a \in C_{1}(T)$. If $a \notin C_{2}\left(C_{1}(T)\right)$ then there is some $y \in C_{1}(T)$ such that $y \succ_{2} a$. Let $b$ be a maximal such $y: b \in C_{1}(T), b \succ_{2} a$, and for any $z \in C_{1}(T) b \succeq_{2} z$. By CR1, $a \sim_{2} C(T)$, and by CR7 we know that $\succeq_{2}$ is transitive, so $b \succ_{2} C(T)$. Now CR1-CR4 construct a partial ordering $\succeq_{2}$ that is complete with respect to all $x, C(T)$ pairs where $x \in T \backslash C(T)$ : for all $x, T$ such that $x \in T \backslash C(T)$, either $x \succeq_{2} C(T)$ or $C(T) \succ_{2} x$. Further, CR6 guarantees that if $x \in T \backslash C(T)$ and $x \succeq_{2} C(T)$ then $x \notin C_{1}(T)$. Now $b \in C_{1}(T)$ and $b \succeq_{2} C(T)$ so, by CR6 (given the use of CR1-CR4), $b \in C(T)$. But if $b \in C(T)$ then, by CR1, $b \sim_{2} C(T)$. But $b \succ_{2} C(T)$ and, by our initial supposition, the constructed ordering $\succeq_{2}$ is acyclic. So it cannot be that $b \sim_{2} C(T)$. That is, $b \notin C(T)$. More importantly, there can be no maximal $y$ such that $y \in C_{1}(T)$ and $y \succ_{2} a$. But $U$ is finite, ${ }^{13}$ so it must instead be the case that, if $a \in C_{1}(T)$, then $a \in C_{2}\left(C_{1}(T)\right)$. And hence if $a \in C(T)$ then $a \in C_{2}\left(C_{1}(T)\right)$.

\footnotetext{
${ }^{13}$ This is assumed throughout this paper. cf. Section 3
} 
In the opposite direction suppose $a \notin C(T)$. If $a \notin T$ or $a \notin C_{1}(T)$ then, trivially, $a \notin C_{2}\left(C_{1}(T)\right)$. Suppose instead that $a \in C_{1}(T)$. So $a \in C_{1}(T) \backslash C(T)$. As discussed in the first part of this proof, CR1-CR4 construct a partial ordering $\succeq_{2}$ that is complete with respect to all $x, C(T)$ pairs where $x \in T \backslash C(T)$. So when CR6 is used in concert with CR1-CR4, CR6 guarantees that if $x \in T \backslash C(T)$ and $x \succeq_{2} C(T)$ then $x \notin C_{1}(T)$. This is just to say that we know, when CR1-CR6 are used to construct $\succeq_{2}$, that if $x \in T \backslash C(T)$ and $x \in C_{1}(T)$, then it is not the case that $x \succeq_{2} C(T)$. Now $a \in C_{1}(T) \backslash C(T)$ and CR1-CR6 are being used, so it cannot be the case that $a \succeq_{2} C(T)$. And so, by the completeness of $\succeq_{2}$ with respect to all $x, C(T)$ pairs where $x \in T \backslash C(T)$, it must instead be the case that $a \prec_{2} C(T)$. But since $C(T) \subseteq C_{1}(T)$ (by CR5), it also follows that $a \notin C_{2}\left(C_{1}(T)\right)$.

So if CR1-CR8 are used to construct acyclic orderings, then $x \in C(T)$ if and only if $x \in C_{2}\left(C_{1}(T)\right)$, for all $T \subseteq U$. CR1-CR8 are a success.

\subsection{Soundness for $\mathrm{TT} \alpha, \mathrm{TTIC}(1)$ and $\mathrm{TTIC}(2)$}

Recall the theorem we are trying to prove:

Theorem 1. $C$ is two-tier rationalizable if and only if $C$ satisfies conditions $T T \alpha, T T I C(1)$ and $T T I C(2)$.

So far we have all the pieces we need to show the theorem to be true in the backwards direction. What remains is to put those pieces together, and to show that if $C$ is twotier rationalizable then $C$ will satisfy conditions $\operatorname{TT} \alpha$, TTIC $(1)$ and $\operatorname{TTIC}(2)$. In this section I provide proofs for this claim.

\subsubsection{Property $\mathrm{TT} \alpha$}

\section{Proposed Condition 1: TT $\alpha$}

For any $x, y \in U$ and $T, S \subseteq U$ :

(a) If $S_{T}(C(T), x)$ then $\neg G_{T}(x, C(T))$.

(b) If $S_{T}(C(T), x), S_{T}(C(S), y)$, and $G_{T}(x, C(S))$ then $\neg G_{T}(y, C(T))$.

Proposition 2. If $C$ is a two-tier choice function, then $C$ will satisfy TT $\alpha$. 
Proof. Suppose $C$ is a two-tier choice function. For (a), let $S_{T}(C(T), a)$. Then there is some $b \in U$ such that $S(C(T), b)$ and $G_{T}(b, a)$. That is, there is some $b \in U$ and $S \subseteq U$ such that $b \in C(S) \cap T \backslash C(T), T \subseteq S$, and $G_{T}(b, a)$. By Lemma $3, C(T) \succ_{2} b$. By Lemmas 1 and 2 and the transitivity of $\succeq_{2}, b \succeq_{2} a$. So, by the transitivity of $\succeq_{2}, C(T) \succ_{2} a$. If $G_{T}(a, C(T))$ then, by Lemmas 1 and 2 and the transitivity of $\succeq_{2}$, $a \succeq_{2} C(T)$. But $\succeq_{2}$ is acyclic, so $\neg G_{T}(a, C(T))$.

For Part (b), let $S_{T}(C(T), a), S_{T}(C(S), b)$, and $G_{T}(a, C(S))$. Following the same reasoning as for (a), this means that $C(T) \succ_{2} a, C(S) \succ_{2} b$, and $a \succeq_{2} C(S)$. By the transitivity of $\succeq_{2}$, then, $C(T) \succ_{2} b$. If $G_{T}(b, C(T))$ then, as above, $b \succeq_{2} C(T)$. But $\succeq_{2}$ is acyclic, so $\neg G_{T}(b, C(T))$.

\subsubsection{Property TTIC(1)}

\section{Proposed Condition 2: TTIC(1)}

(a) For any $x \in U, T \subseteq U$ : If $x \in T \backslash C(T)$ then $\exists y \in C(T) \backslash\{x\}$.

(b) For any sequence of sets $T_{1}, \ldots, T_{n} \subseteq U$, if there is a corresponding sequence of elements $x_{1}, \ldots, x_{n} \in U[n \geq 2]$ such that:

$$
\begin{aligned}
& x_{1} \in T_{1} \backslash C\left(T_{1}\right) \text {, and } \\
& x_{i} \in\left(\left(T_{1} \cup \ldots \cup T_{i-1}\right) \cap T_{i}\right) \backslash\left(C\left(T_{i}\right) \cup\left\{x_{1}, \ldots, x_{i-1}\right\}\right)[\text { for } i=2, \ldots, n], \\
& \text { and } \\
& G_{T}\left(x_{i}, C\left(T_{i}\right)\right) \text { or } S_{T}\left(x_{i}, C\left(T_{i}\right)\right)[\text { for } i=1, \ldots, n],
\end{aligned}
$$

then $\exists y \in\left(T_{1} \cup \ldots \cup T_{n}\right) \backslash\left\{x_{1}, \ldots, x_{n}\right\}$.

Proposition 3. If $C$ is a two-tier choice function then $C$ will satisfy TTIC(1).

Proof. Part (a) is trivial: since $C(T) \neq \emptyset$ and $x \notin C(T)$, then $\exists y \in C(T) \backslash\{x\}$. For part (b) suppose that $C$ is a two-tier choice function and $T_{1}, \ldots, T_{n} \subseteq U[n \geq 2]$. Let $a_{1}, \ldots, a_{n} \in U$ be such that $a_{1} \in T_{1} \backslash C\left(T_{1}\right), a_{i} \in\left(\left(T_{1} \cup \ldots \cup T_{i-1}\right) \cap T_{i}\right) \backslash C\left(T_{i}\right)$ [for $i=2, \ldots, n]$, and, for each $i=1, \ldots, n$, either $G_{T}\left(a_{i}, C\left(T_{i}\right)\right)$ or $S_{T}\left(a_{i}, C\left(T_{i}\right)\right)$. We want to show that there is some $y \in\left(T_{1} \cup \ldots \cup T_{n}\right) \backslash\left\{a_{1}, \ldots, a_{n}\right\}$. Suppose to the contrary that $T_{1} \cup \ldots \cup T_{n}=\left\{a_{1}, \ldots, a_{n}\right\}$, and let $a_{j} \in C\left(T_{1} \cup \ldots \cup T_{n}\right)$ [for some $\left.j=1, \ldots, n\right]$. Now $a_{j} \in C\left(T_{1} \cup \ldots \cup T_{n}\right)$ and $C$ is two-tier, so $a_{j} \in C_{1}\left(T_{1} \cup \ldots \cup T_{n}\right)$. By Property $\alpha$, then, $a_{j} \in C_{1}\left(T_{j}\right)$. But $a_{j} \notin C\left(T_{j}\right): a_{j} \in C\left(T_{1} \cup \ldots \cup T_{n}\right) \cap T_{j} \backslash C\left(T_{j}\right)$ and $T_{j} \subseteq\left(T_{1} \cup \ldots \cup T_{n}\right)$. So it must be the case, by Lemma 3 , that $C\left(T_{j}\right) \succ_{2} a_{j}$. Now $G_{T}\left(a_{j}, C\left(T_{j}\right)\right)$ if and only if $\exists b_{j} \in U$ such that $G_{T}\left(a_{j}, b_{j}\right)$ and $I\left(\left\{b_{j}\right\} \cup C\left(T_{j}\right)\right)$. By Lemmas 1 and 2 and the 
transitivity of $\succeq_{2}$, then, $G_{T}\left(a_{j}, C\left(T_{j}\right)\right)$ if and only if $a_{j} \succeq_{2} C\left(T_{j}\right)$. But $C\left(T_{j}\right) \succ_{2} a_{j}$, so $\neg G_{T}\left(a_{j}, C\left(T_{j}\right)\right)$. Rather, $S_{T}\left(a_{j}, C\left(T_{j}\right)\right)$. But by the definition of $S_{T}\left(a_{j}, C\left(T_{j}\right)\right)$, Lemmas $1,2, \& 3$, and the transitivity of $\succeq_{2}, S_{T}\left(a_{j}, C\left(T_{j}\right)\right)$ if and only if $a_{j} \succ_{2} C\left(T_{j}\right)$. So it cannot be that $C\left(T_{j}\right) \succ_{2} a_{j}$. And so it cannot be that $a_{j} \in C\left(T_{1} \cup \ldots \cup T_{n}\right)$, for any $j=1, \ldots, n$. That is, $\exists y \in C\left(T_{1} \cup \ldots \cup T_{n}\right) \backslash\left\{a_{1}, \ldots, a_{n}\right\}$. More to the point, $\exists y \in\left(T_{1} \cup \ldots \cup T_{n}\right) \backslash\left\{a_{1}, \ldots, a_{n}\right\}$.

\subsubsection{Property TTIC(2)}

\section{Proposed Condition 3: TTIC(2)}

(a) For any $S, T \subseteq U$ : if $x \in C(S) \cap T \backslash C(T)$ and either $G_{T}(x, C(T))$ or $S_{T}(x, C(T))$, then $\exists y \in T \backslash S$.

(b) For any sequence of sets $S, T_{1}, \ldots, T_{n} \subseteq U[n \geq 2]$, if there is a corresponding sequence of elements $x_{1}, \ldots, x_{n}$ such that

$$
\begin{aligned}
& x_{1} \in C(S) \cap T_{1} \backslash C\left(T_{1}\right), \text { and } \\
& x_{i} \in\left(\left(T_{1} \cup \ldots \cup T_{i-1}\right) \cap T_{i}\right) \backslash\left(C\left(T_{i}\right) \cup S \cup\left\{x_{1}, \ldots, x_{i-1}\right\}\right)[i=2, \ldots, n] \\
& \text { and } \\
& G_{T}\left(x_{i}, C\left(T_{i}\right)\right) \text { or } S_{T}\left(x_{i}, C\left(T_{i}\right)\right)[\mathrm{i}=1, \ldots, \mathrm{n}]
\end{aligned}
$$

then $\exists y \in\left(T_{1} \cup \ldots \cup T_{n}\right) \backslash\left(S \cup\left\{x_{2}, \ldots, x_{n}\right\}\right)$.

Proposition 4. If $C$ is a two-tier choice function then $C$ satisfies TTIC(2).

Proof. Let $C$ be a two-tier choice function. For part (a), let $a \in C(S) \cap T \backslash C(T)$ and suppose $G_{T}(a, C(T))$ or $S_{T}(a, C(T))$. By Lemmas 1,2 and 3 and the transitivity of $\succeq_{2}$, this implies that $a \succeq_{2} C(T)$. So $a \in T \backslash C(T)$ and $a \succeq_{2} C(T)$; by Lemma 4, $a \notin C_{1}(T)$. That is to say that for any first-tier ordering $\succeq$, there is some $y \in T \backslash\{a\}$ such that $y \succ a$. Now $a \in C(S)$, so $a \in C_{1}(S)$, and hence there is some first-tier ordering $\succeq_{S}$ such that $\forall z \in S a \succeq_{S} z$. And so it follows that there is a first-tier ordering $\succeq_{S}$ such that, for some $y \in T \backslash\{a\}, y \succ_{S} a \succeq_{S} S$. Since $\succeq_{S}$ is acyclic and transitive it follows that $y \in T \backslash S$. And hence there is some $y \in T \backslash S$.

For part (b), let $a_{1} \in C(S) \cap T_{1} \backslash C\left(T_{1}\right), a_{i} \in\left(\left(T_{1} \cup \ldots \cup T_{i-1}\right) \cap T_{i}\right) \backslash\left(C\left(T_{i}\right) \cap S \cap\right.$ $\left.\left\{a_{1}, \ldots, a_{i-1}\right\}\right)[i=2, \ldots, n]$, and $G_{T}\left(a_{i}, C\left(T_{i}\right)\right)$ or $S_{T}\left(a_{i}, C\left(T_{i}\right)\right)$ for all $i=1, \ldots, n$. From the proof of Proposition 3 above we know that $C\left(T_{1} \cup \ldots \cup T_{n}\right) \cap\left\{a_{1}, \ldots, a_{n}\right\}=\emptyset$. But suppose $\left(T_{1} \cup \ldots \cup T_{n}\right) \subseteq\left(S \cup\left\{a_{1}, \ldots, a_{n}\right\}\right)$. Then $C\left(T_{1} \cup \ldots \cup T_{n}\right) \subseteq S$. Now $a_{1} \in C(S)$, so $a_{1} \in C_{1}(S)$ : Let $\succeq_{S}$ be a first-tier ordering where $a_{1} \succeq_{S} S$. Since $C\left(T_{1} \cup \ldots \cup T_{n}\right) \subseteq S$ 
and $a_{1} \succeq_{S} S$, then $a_{1} \succeq_{S} C\left(T_{1} \cup \ldots \cup T_{n}\right)$. By (a) there is some $y \in T_{1} \backslash S$ such that $y \succ_{S} a_{1}$. Since $\left(T_{1} \cup \ldots \cup T_{n}\right) \subseteq\left(S \cup\left\{a_{2}, \ldots, a_{n}\right\}\right)$ then there is some $y \in\left\{a_{2}, \ldots, a_{n}\right\}$ such that $y \succ_{S} a_{1}$; and there is an optimal such $y$ : there is a $y \in\left\{a_{2}, \ldots, a_{n}\right\}$ such that $y \succ_{S} a_{1}$ and, for any $a_{k} \in\left\{a_{2}, \ldots, a_{n}\right\}, y \succeq_{S} a_{k}$. Let $a_{j}$ be that element. So $a_{j} \succeq_{S} a_{k}$ for all $a_{k} \in\left\{a_{2}, \ldots, a_{n}\right\}$ and $a_{j} \succ_{S} a_{1} \succeq_{S} S$. And hence $a_{j} \in C_{1}\left(S \cup\left\{a_{2}, \ldots, a_{n}\right\}\right)$. By Property $\alpha$, then, since $T_{j} \subseteq\left(T_{1} \cup \ldots \cup T_{n}\right) \subseteq\left(S \cup\left\{a_{2}, \ldots, a_{n}\right\}\right), a_{j} \in C_{1}\left(T_{j}\right)$. So $a_{j} \in$ $C_{1}\left(T_{j}\right) \backslash C\left(T_{j}\right)$, and hence, by Lemma $4, C\left(T_{j}\right) \succ_{2} a_{j}$. That is to say, $\neg G_{T}\left(a_{j}, C\left(T_{j}\right)\right)$ and $\neg S_{T}\left(a_{j}, C\left(T_{j}\right)\right.$ ), contrary to supposition (this follows from Lemmas 1-3 and the transitivity of $\left.\succeq_{2}\right)$. So it must not be the case that $\left(T_{1} \cup \ldots \cup T_{n}\right) \subseteq\left(S \cup\left\{a_{2}, \ldots, a_{n}\right\}\right)$. That is, $\exists y \in\left(T_{1} \cup \ldots \cup T_{n}\right) \backslash\left(S \cup\left\{a_{2}, \ldots, a_{n}\right\}\right)$.

\subsection{Putting it All Together}

And now we have all the pieces we need to prove our main theorem.

Theorem 1. $C$ is two-tier rationalizable if and only if $C$ satisfies conditions $\mathrm{TT} \alpha, \operatorname{TTIC}(1)$ and $\operatorname{TTIC}(2)$.

Proof. The forward direction follows from Propositions 2, 3 and 4: If $C$ is a twotier choice function then $C$ satisfies $\operatorname{TT} \alpha, \operatorname{TTIC}(1)$, and $\operatorname{TTIC}(2)$. In the opposite direction we know that if CR1-CR8 are used to produce acyclic orderings then $C(S)=C_{2}\left(C_{1}(S)\right)$ for all $S \subseteq U$ (by Proposition 1). By Lemma 5, CR1-CR3 produce an acyclic partial order $\succeq_{2}$ provided $C$ satisfies TT $\alpha$. By Lemma $6, x \succeq_{2} C(T)$ for this order if and only if $G_{T}(x, C(T))$ or $S_{T}(x, C(T))$. CR4 sets $C(T) \succ_{2} x$ only when $\neg G_{T}(x, C(T))$ and $\neg S_{T}(x, C(T))$ (and $x \in T \backslash C(T)$ ); and CR7 closes this revised $\succeq_{2}$ under transitivity. So CR4 and CR7 will not introduce cycles into the constructed partial order. And finally, CR8 completes the ordering in such a way that nothing untoward occurs: a relation between pairs of unrelated elements is established, and then the relation is closed under transitivity before a second unrelated pair is identified. So, if $C$ satisfies TT $\alpha$ and CR1-CR 8 are used to construct $V_{2}(U)$, then the constructed ordering $\succeq_{2}$ will be acyclic.

In the first tier, the potential for cycles arises with the use of CR6 (because that is where strict relations are introduced). But if $C$ satisfies TTIC(1) and TTIC(2), as discussed at length above, then CR6 can be completed without producing cycles. And, again, CR7 and CR8 will not introduce any cycles. So if a choice function $C$ satisfies TT $\alpha$, TTIC(1) and TTIC (2) then CR1-CR8 can be used to produce acyclic orderings such that $C(S)=C_{2}\left(C_{1}(S)\right)$ for all $S \subseteq U$. That is, if $C$ satisfies TT $\alpha$, TTIC $(1)$ and TTIC $(2)$, then $C$ is two-tier rationalizable. 


\section{Conclusion}

At the beginning of this paper I set out to characterize a version of Isaac Levi's multitiered decision rule. The version in question involves two tiers, with a single ordering in the second tier, and uses an ordinal framework instead of the cardinal one that Levi would no doubt prefer.

These modifications were made in order to simplify the demands of the project: the characterization in this paper can be understood as a first step toward characterizing Levi's full blown rule. It should also be noted that Levi works with convex sets of (cardinal) utilities - an assumption that my result does not require. In this dimension it might be argued that my result is more general than Levi's full blown rule. ${ }^{14}$

Regardless, the main insight provided by the characterization of two-tier admissibility in this paper has to do with the interplay between tiers for a two-tier choice function. Additionally, when Property $\alpha$ is violated there are specific constraints on the second tier ordering: If $x \in C(S) \cap T \backslash C(T)$ and $T \subseteq S$, then $C(T) \succ_{2} x$.

One weakness in this paper is that many of the proofs are in their 'negative' form, utilizing the heuristic of proof by contradiction. This is due largely to the complexity that would be required to spell out all possible configurations of $V_{1}(U)$ and $V_{2}(U)$ associated with features of two-tier admissibility. But it also means that while some insight has been gained into the nature of two-tier choice functions, there is more insight to be had.

In line with this observation it should be noted that the current version of CR6 is more complicated than one might like. Greater insight into the nature of two-tier choice functions might make way for a simpler specification of CR6. However, at this juncture no such simplification presents itself.

One suggestion for such a simplification is this: ${ }^{15}$

For each $\succeq_{S} \in V_{1}(U)$ : If $x \in T \backslash C(T)$ and $x \succeq_{2} C(T)$ then, for all $y \in T \backslash\{x\}$ such that

(1) $x \in C(S)$ if and only if $y \notin S$, and

(2) $x \notin C(S \cup\{x, y\})$, and

(3) $\neg \exists T^{\prime}$ such that $y \in C\left(T^{\prime}\right)$ and $y \succeq_{2} C\left(T^{\prime}\right)$,

set $y \succ_{S} x$.

\footnotetext{
${ }^{14}$ Thanks to Teddy Seidenfeld for pointing this out.

${ }^{15}$ Thanks to Horacio Arlo-Costa for this suggestion.
} 
This is an intriguing suggestion, and it will certainly not produce cyclic orderings, but it will only suffice as CR6 if the following lemma is true:

Lemma 7. For every $x \in T \backslash C(T)[T \subseteq U]$ where $G_{T}(x, C(T))$ or $S_{T}(x, C(T))$ holds, there is some $y \in T \backslash\{x\}$ such that

(1) $x \in C(S)$ if and only if $y \notin S$, and

(2) $x \notin C(S \cup\{x, y\})$, and

(3) $\neg \exists T^{\prime}$ such that $y \in C\left(T^{\prime}\right)$ and $y \succeq_{2} C\left(T^{\prime}\right)$.

If no such $y \in T \backslash\{x\}$ exists, then CR6 will not pick some 'appropriate' $y$ and set $y \succ_{S} x$, thereby blocking $x$ from admissibility in $C_{1}(T)$. That is, CR6 will not do the job it is supposed to do.

But showing this lemma to be true - or cooking up a two-tier rationalizable counterexample - is not straightforward. Part (1) follows from TTIC(1); but showing that there is always some $y \in T \backslash\{x\}$ that satisfies (1), (2) and (3) is more difficult. As a result, a proof (or counter-example) for this proposal goes beyond the scope of this paper.

An additional directive for future work is obvious: In order to do Levi's proposal justice we need to be able to characterize his multi-tier decision rule with the use of cardinal preference relations instead of ordinal ones. The point of this paper was simply to lay a foundation for that future work. By treating a limited version of Levi's rule I have taken the first step in what should be thought of as a much larger project.

Nevertheless, that foundation has been laid: a choice function $C$ is two-tier rationalizable if and only if $C$ satisfied $\operatorname{TT} \alpha, \operatorname{TTIC}(1)$, and $\operatorname{TTIC}(2)$. 


\section{References}

Aizerman, M.A. and Malishevsky, A.V. (1981). General Theory of Best Variant Choices. IEEE Trans. Automatic Control. AC-26, 1030-1040.

Chernoff, H. (1954). Rational Selection of Decision Functions. Econometrica. 22, 422-443.

Levi, Isaac (1986). Hard Choices: Decision making under unresolved conflict. New York: Cambridge University Press.

Moulin, H. (1985) Choice Functions Over a Finite Set: A Summary. Social Choice and Welfare. 2, 147-160

Sen, A. (1969). Quasi-transitivity, Rational Choice and Collective Decisions. Review of Economic Studies. 36, 381-393.

Sen, A. (1971). Choice Functions and Revealed Preference. Review of Economic Studies. 38, 307-317. 